\title{
Discontinuous Galerkin methods for fractional elliptic problems
}

\author{
Tarek Aboelenen \\ Department of Mathematics, Assiut University, Assiut 71516, Egypt
}

\begin{abstract}
We provide a mathematical framework for studying different versions of discontinuous Galerkin (DG) approaches for solving 2D Riemann-Liouville fractional elliptic problems on a finite domain. The boundedness and stability analysis of the primal bilinear form are provided. A priori error estimate under energy norm and optimal error estimate under $L^{2}$ norm are obtained for DG methods of the different formulations. Finally, the performed numerical examples confirm the optimal convergence order of the different formulations.
\end{abstract}

Keywords: fractional elliptic problems, discontinuous Galerkin methods, continuity, coercivity, optimal convergence.

AMS subject classifications: 26A33, 35R11, 65M60, 65M12.

\section{Introduction}

The fractional differential operators, as a natural generalization of the concept of classical operators of integer orders to fractional orders, has become more popular in science and engineering such as fractals [1], kinetic theories of systems with chaotic dynamics [2, 3, 4, pseudochaotic dynamics [5], anomalous transport [6], viscoelastic materials [7], electrochemistry [8] and image processing [9], etc.

Unlike the classical partial differential equations (PDEs), there is more difficult to find the analytical solutions of the fractional partial differential equations (FPDEs) explicitly. Therefore, it is necessary to use numerical methods. For the existence and analytical solutions to FPDEs [10, 11, 12, 13] and references therein. Many powerful methods have been proposed for numerically solving the FPDEs, e.g., finite element methods [14, 15, finite difference methods [16, 17, 18, 19, spectral methods [20, 21, 22, 23] and DG methods [24, 25, 26, 27, 28, 29, 30, 31] and so many others.

Recently, Jin et al. 32] proved the existence and uniqueness of a weak solution to the space-fractional parabolic equation using finite element method; they showed an enhanced regularity of the solution and derived the error estimate for both semidiscrete and fully discrete solution. Wang and Yang [33] generalized the analysis to the case of fractional elliptic problems with variable coefficient, analyzed the regularity of the solution in Hölder

Email address: tarek.aboelenen@aun.edu.eg (Tarek Aboelenen) 
spaces, and established the well-posedness of a Petrov-Galerkin formulation. In [34, the authors constructed a Petrov-Galerkin spectral element method to solve the weak form of fractional elliptic problems.

The Discontinuous Galerkin (DG) method is famous for high accuracy properties and extreme flexibility [35, 36, 37, 38. There exist many applications of DG methods to solve FPDEs in one dimension, for example, fractional convection-diffusion equations [28, 39, time fractional diffusion and wave equations [24, 25, 26, 27, nonlinear Riesz space fractional Schrödinger type equations [30, 39], fractional Cahn-Hilliard equation [40] and distributed-order time and space-fractional convection-diffusion and Schrödinger type equations [31]. In the two dimensional case, Ji and Tang [41] have applied the DG methods to recast the fractional diffusion equations in rectangular meshes. Qiu et al. 42] proposed a nodal DG methods for two dimensional fractional diffusion equations on unstructured meshes. They proved stability and optimal order of convergence $N+1$ for the fractional diffusion problem in triangular meshes.

There are several DG methods for solving elliptic and parabolic problems. For examples, the interior penalty (IP) methods [43, 44, 45, 46, 47, the nonsymmetric interior penalty Galerkin (NIPG) methods [48, 49], unified analysis of discontinuous methods [50] and a compact discontinuous Galerkin (CDG) method [51]. Recent developments of DG methods on elliptic problems include the hybridized DG method [52], the over penalized DG method [53] and the weak Galerkin method [54, etc. To the best of our knowledge, however, the DG methods, which is an important approach to solve PDEs and FPDEs, have not been considered for the fractional elliptic problems. Thus, we dedicate this work to investigate the fractional elliptic problems in triangular meshes by using DG methods. We shall consider two dimensional fractional elliptic problems in triangular meshes

$$
-\frac{\partial^{\alpha} u(\boldsymbol{x})}{\partial x^{\alpha}}-\frac{\partial^{\beta} u(\boldsymbol{x})}{\partial y^{\beta}}=f(\boldsymbol{x}), \boldsymbol{x}=(x, y) \in \mathcal{R}^{2},
$$

with homogeneous boundary conditions. $\frac{\partial^{\alpha}}{\partial x^{\alpha}}, \frac{\partial^{\beta}}{\partial y^{\beta}}, \alpha, \beta \in(1,2]$ refer to the Riemann-Liouville (R-L) fractional derivatives and $f(\boldsymbol{x})$ is a source term. Notice that the assumption of homogeneous boundary conditions is for the convenience of the theoretical analysis only and is not essential.

The rest of the article is organized as follows. In section 2, we reminder Some useful definitions and results of fractional calculus. In section 3 we present scheme formulations of DG methods, and in section 4 , we relate the conservativity and consistency properties of the numerical fluxes and the consistency and adjoint consistency properties of the bilinear form of the primal formulation. We perform the boundedness, stability and convergence analysis for the two dimensional fractional elliptic problems in section 5 . We present some numerical examples showing the optimality of our theoretical results and illustrate the flexibility and efficiency of the schemes in section 6. Finally, the concluding remarks are given.

\section{Preliminaries}

We first introduce the definitions of fractional derivatives and integrals [55] and review a few lemmas for our analysis. 


\subsection{Liouville fractional calculus}

The right-sided and left-sided R-L integrals of order $\mu$, when $0<\mu<1$, for the function $f(x)$ is defined, respectively, as

$$
\left({ }_{-\infty} \mathcal{I}_{x}^{\mu} f\right)(x)=\frac{1}{\Gamma(\mu)} \int_{-\infty}^{x} \frac{f(s) d s}{(x-s)^{1-\mu}}, \quad x>-\infty
$$

and

$$
\left({ }_{x} \mathcal{I}_{\infty}^{\mu} f\right)(x)=\frac{1}{\Gamma(\mu)} \int_{x}^{\infty} \frac{f(s) d s}{(s-x)^{1-\mu}}, \quad x<\infty
$$

The right and left R-L fractional derivatives of function $f$ are defined by

$$
\left({ }_{-\infty} \mathcal{D}_{x}^{\mu} f\right)(x)=\frac{1}{\Gamma(n-\mu)}\left(\frac{d}{d x}\right)^{n} \int_{-\infty}^{x} \frac{f(s) d s}{(x-s)^{-n+1+\mu}}, \quad x>-\infty,
$$

and

$$
\left({ }_{x} \mathcal{D}_{\infty}^{\mu} f\right)(x)=\frac{1}{\Gamma(n-\mu)}\left(\frac{-d}{d x}\right)^{n} \int_{x}^{\infty} \frac{f(s) d s}{(s-x)^{-n+1+\mu}}, \quad x<\infty .
$$

for any $(n-1<\mu<n), n \in \mathbb{N}^{+}$.

Definition 2.1. Let $\alpha>0$. Define the norm

$$
\|u\|_{H^{-\alpha}(\mathcal{R})}:=\left\||\omega|^{-\alpha} \widehat{u}\right\|_{L^{2}(\mathcal{R})}
$$

where $\widehat{u}(\omega)$ is the Fourier transform of $u(x)$ and let $H^{-\alpha}(\mathcal{R})$ denote the closure of $C_{0}^{\infty}(\mathcal{R})$ with respect to $\|\cdot\|_{H^{-\alpha}(\mathcal{R})} \cdot$

\section{Lemma 2.1.}

$$
\left({ }_{-\infty} \mathcal{I}_{x}^{-\alpha} u,{ }_{x} \mathcal{I}_{\infty}^{-\alpha} u\right)=\cos (\alpha \pi)\left\|_{-\infty} \mathcal{I}_{x}^{-\alpha} u\right\|_{L^{2}(\mathcal{R})}^{2}=\cos (\alpha \pi)\|u\|_{H^{-\alpha}(\mathcal{R})}^{2} .
$$

Generally, we consider the problems in a bounded domain and let the domain $\Upsilon=[a, b]$ instead of $\mathbb{R}$.

Definition 2.2. Define the spaces $H_{0}^{-\alpha}(\Upsilon)$ as the closure of $C_{0}^{\infty}(\Upsilon)$.

Theorem 2.1. If $-\alpha_{2}<-\alpha_{1}<0$, then $H_{0}^{-\alpha_{1}}(\Upsilon)$ is embedded into $H_{0}^{-\alpha_{2}}(\Upsilon)$ is embedded into both of them.

Lemma 2.2. (See [10]) The fractional integration operator ${ }_{-\infty} \mathcal{I}_{x}^{-\alpha}$ and ${ }_{x} \mathcal{I}_{\infty}^{-\alpha}$ are bounded in $L^{2}(\Upsilon)$ :

$$
\left\|_{-\infty} \mathcal{I}_{x}^{-\alpha} u\right\|_{L^{2}(\Upsilon)} \leq C\|u\|_{L^{2}(\Upsilon)}
$$

and

$$
\left\|{ }_{x} \mathcal{I}_{\infty}^{-\alpha} u\right\|_{L^{2}(\Upsilon)} \leq C\|u\|_{L^{2}(\Upsilon)}
$$




\section{The DG methods for for fractional elliptic problems}

In this section, we present DG methods for the two-dimensional fractional elliptic problems with homogeneous boundary conditions on the form.

$$
\begin{cases}-\frac{\partial}{\partial x} \mathcal{I}_{x}^{2-\alpha} \frac{\partial}{\partial x} u(\boldsymbol{x})-\frac{\partial}{\partial y_{c}} \mathcal{I}_{y}^{2-\beta} \frac{\partial}{\partial y} u(\boldsymbol{x})=f(\boldsymbol{x}) & \boldsymbol{x} \in \Omega, \\ u(\boldsymbol{x})=0 & \boldsymbol{x} \in \partial \Omega\end{cases}
$$

where $\Omega=(a, b) \times(c, d)$ with boundary $\partial \Omega$. To obtain a high order DG scheme for the fractional derivative, we rewrite it as a composite of a fractional integral and first order derivatives and convert the fractional elliptic problems 2.3 into a system of low order equations. We introduce the auxiliary variables $\boldsymbol{p}=\left(p^{x}, p^{y}\right)$ and $\boldsymbol{q}=\left(q^{x}, q^{y}\right)$, and rewrite as

$$
\begin{cases}-\nabla \cdot \boldsymbol{q}=f(\boldsymbol{x}) & \boldsymbol{x} \in \Omega, \\ \boldsymbol{q}={ }_{L} I_{x}^{\bar{\alpha}} \boldsymbol{p}=\left({ }_{a} \mathcal{I}_{x}^{\alpha_{1}} p^{x},{ }_{c} \mathcal{I}_{y}^{\alpha_{2}} p^{y}\right) & \boldsymbol{x} \in \Omega, \\ \boldsymbol{p}=\nabla u & \boldsymbol{x} \in \Omega, \\ u(\boldsymbol{x})=0 & \boldsymbol{x} \in \partial \Omega .\end{cases}
$$

where ${ }_{L} I_{\boldsymbol{x}}^{\bar{\alpha}}=\left({ }_{a} \mathcal{I}_{x}^{\alpha_{1}},{ }_{{ }^{\prime}} \mathcal{I}_{y}^{\alpha_{2}}\right),\left(\alpha_{1}, \alpha_{2}\right)=(2-\alpha, 2-\beta)$ and $\alpha_{1}, \alpha_{2} \in(0,1)$. Here, we assume that the physical domain $\Omega$ is well approximated by the computational domain $\Omega_{h}$. This is a space filling triangulation composed of a collection of K geometry-conforming nonoverlapping elements $D^{k}$.

To complete the DG schemes, we introduce the local inner products and norms

$$
\int_{\Omega} v u d \boldsymbol{x}=\sum_{k=1}^{K}(v, u)_{D^{k}}, \quad(v, u)_{\Gamma}=\sum_{k=1}^{K}(v, u)_{\partial D^{k}}, \quad\|v\|_{\Omega, h}^{2}=\sum_{k=1}^{K}\|v\|_{D^{k}}, \quad\|v\|_{D^{k}}=\int_{D^{k}} v^{2} d \boldsymbol{x} .
$$

The associated Sobolev norms are defined as

$$
\|v\|_{\Omega, q}^{2}=\sum_{|\gamma|=0}^{q}\left\|v^{(\gamma)}\right\|_{\Omega}, \quad\|v\|_{\Omega, q, h}^{2}=\sum_{k=1}^{K}\|v\|_{D^{k}, q}, \quad\|v\|_{D^{k}, q}^{2}=\sum_{|\gamma|=0}^{q}\left\|v^{(\gamma)}\right\|_{D^{k}}
$$

We define the space of functions, $v \in H^{\gamma}(\Omega)$, as those functions for which $\|v\|_{\Omega, q}$ or $\|v\|_{\Omega, q, h}$ is bounded. We will need the semi-norms

$$
|v|_{\Omega, q, h}^{2}=\sum_{k=1}^{K}|v|_{D^{k}, q}^{2}, \quad|v|_{D^{k}, q}^{2}=\sum_{|\gamma|=q}\left\|v^{(\gamma)}\right\|_{D^{k}}^{2}
$$

Here, $(\Omega, h)$ reflects that $\Omega$ is only approximated by the union of $D^{k}$, that is

$$
\Omega \simeq \Omega_{h}=\bigcup_{k=1}^{K} D^{k}
$$

and $\Gamma_{b}$ denotes the set of external edges, the set of unique purely internal edges $\Gamma_{i}$ and $\Gamma$ denotes the union of the boundaries of the elements $D^{k}$ of $\Omega_{h}$ and $\Gamma=\Gamma_{i} \bigcup \Gamma_{b}$. 
For $e \in \Gamma$, we refer to the interior information of the element by a superscript '-' and to the exterior information by a superscript ' + '. Using this notation, it is useful to define the jump and the average operators are given as

$$
\begin{gathered}
\llbracket v \rrbracket=\mathbf{n}^{+} v^{+}+\mathbf{n}^{-} v^{-}, \llbracket \mathbf{v} \rrbracket=\mathbf{n}^{+} \cdot \mathbf{v}^{+}+\mathbf{n}^{-} \cdot \mathbf{v}^{-} \quad \text { on } e \in \Gamma_{i}, \llbracket v \rrbracket=\mathbf{n} v, \llbracket \mathbf{v} \rrbracket=\mathbf{n} \cdot \mathbf{v} \quad \text { on } e \in \Gamma_{b} . \\
\{v\}=\frac{u^{+}+v^{-}}{2} \quad \text { on } e \in \Gamma_{i},\{v\}=v \quad \text { on } e \in \Gamma_{b},
\end{gathered}
$$

where $\mathbf{n}$ is the outward unit normal.

For any real number $s$, the broken Sobolev space is defined as

$$
H^{s}\left(\Omega_{h}\right)=\left\{v \in L^{2}(\Omega)|v|_{D^{k}} \in H^{s}\left(D^{k}\right), k=1,2, \cdots, K\right\} .
$$

When $s=0$, we denote $H^{0}\left(\Omega_{h}\right)=L^{2}\left(\Omega_{h}\right)$ as general. In addition, we define the finite dimensional subspace of $H^{1}\left(\Omega_{h}\right)$ as

$$
V_{h}=\left\{v: \Omega_{h} \rightarrow \mathbb{R}|v|_{D^{k}} \in P_{N}^{2}\left(D^{k}\right), k=1,2, \cdots, K\right\} .
$$

Now we define the weak formulation with which our DG methods. We multiply the first, second, and the third equation of 3.2 by arbitrary, smooth test functions $v, \boldsymbol{\phi}$ and $\boldsymbol{\pi}$, respectively, and integrate by parts, we obtain

$$
\begin{aligned}
(\boldsymbol{q}, \nabla v)_{D^{k}}-(\mathbf{n} \cdot \boldsymbol{q}, v)_{\partial D^{k}} & =(f, v)_{D^{k}} \\
(\boldsymbol{q}, \boldsymbol{\phi})_{D^{k}} & =\left({ }_{L} I_{x}^{\bar{\alpha}} \boldsymbol{p}, \boldsymbol{\phi}\right)_{D^{k}} \\
(\boldsymbol{p}, \boldsymbol{\pi})_{D^{k}} & =(u, \mathbf{n} \cdot \boldsymbol{\pi})_{\partial D^{k}}-(u, \nabla \cdot \boldsymbol{\pi})_{D^{k}}
\end{aligned}
$$

where $(u, \boldsymbol{p}, \boldsymbol{q}) \in H^{1}\left(\Omega_{h}\right) \times\left(L^{2}\left(\Omega_{h}\right)\right)^{2} \times\left(H^{1}\left(\Omega_{h}\right)\right)^{2}$ and test functions $v \in L^{2}\left(\Omega_{h}\right), \boldsymbol{\phi}=\left(\phi^{x}, \phi^{y}\right), \boldsymbol{\pi}=\left(\pi^{x}, \pi^{y}\right) \in$ $\left(H^{1}\left(\Omega_{h}\right)\right)^{2}=H^{1}\left(\Omega_{h}\right) \times H^{1}\left(\Omega_{h}\right)$.

In order to derive the primal form of our DG schemes, we first define $u_{h}, \boldsymbol{p}_{h}, \boldsymbol{q}_{h}$ as the approximation of $u, \boldsymbol{p}, \boldsymbol{q}$ and then restrict the trial and tests functions $v$ to $V_{h}, \boldsymbol{\phi}, \boldsymbol{\pi}$ to $\left(V_{h}\right)^{2}=V_{h} \times V_{h}$. Our final purpose is to find $\left(u_{h}, \boldsymbol{p}_{h}, \boldsymbol{q}_{h}\right) \in V_{h} \times\left(V_{h}\right)^{2} \times\left(V_{h}\right)^{2}$ such that for all $v \in V_{h}, \boldsymbol{\pi}, \boldsymbol{\phi} \in\left(V_{h}\right)^{2}$ the following holds:

$$
\begin{aligned}
\left(\boldsymbol{q}_{h}, \nabla v\right)_{D^{k}}-\left(\mathbf{n} \cdot \widehat{\boldsymbol{q}}_{h}, v\right)_{\partial D^{k}} & =(f, v)_{D^{k}} \\
\left(\boldsymbol{q}_{h}, \boldsymbol{\phi}\right)_{D^{k}} & =\left({ }_{L} I_{x}^{\bar{\alpha}} \boldsymbol{p}_{h}, \boldsymbol{\phi}\right)_{D^{k}}, \\
\left(\boldsymbol{p}_{h}, \boldsymbol{\pi}\right)_{D^{k}} & =\left(\widehat{u}_{h}, \mathbf{n} \cdot \boldsymbol{\pi}\right)_{\partial D^{k}}-\left(u_{h}, \nabla \cdot \boldsymbol{\pi}\right)_{D^{k}}
\end{aligned}
$$

The choice of the numerical fluxes $\widehat{u}_{h}$ and $\widehat{\boldsymbol{q}}_{h}$ is quite delicate, as it can affect the accuracy of the method and the stability [56, 57, 58, We must define the numerical fluxes $\widehat{u}_{h}$ and $\widehat{\boldsymbol{q}}_{h}$ carefully. So, we adopt numerical fluxes as defined; see Table 1.

\section{Primal forms, consistency, conservation}

In this section, we prove conservation and consistency the numerical fluxes properties are reflected in consistency and adjoint consistency of the primal formulation. 


\begin{tabular}{||c||c|c||}
\hline & $\widehat{u}$ & $\widehat{\boldsymbol{q}}$ \\
\hline Central flux & $\{u\}$ & $\{\boldsymbol{q}\}-\lambda \llbracket u \rrbracket$ \\
LDG flux & $\{u\}+\eta \cdot \llbracket u \rrbracket$ & $\{\boldsymbol{q}\}-\eta \llbracket \boldsymbol{q} \rrbracket-\lambda \llbracket u \rrbracket$ \\
IP flux & $\{u\}$ & $\left\{{ }_{L} I_{\boldsymbol{x}}^{\overline{\boldsymbol{\alpha}}}(\nabla u)\right\}-\lambda \llbracket u \rrbracket$ \\
\hline
\end{tabular}

Table 1: The Central, LDG and IP fluxes.

To obtain a better understanding of the different schemes, we try to eliminate $\boldsymbol{p}_{h}$ and $\boldsymbol{q}_{h}$, to obtain the primal form in terms of only $u_{h}$. To do that, we introduce the following result:

Lemma 4.1. Assume that $\Omega$ has been triangulated into $K$ elements, $D^{k}$, then

$$
\sum_{k=1}^{K}(\boldsymbol{n} \cdot \boldsymbol{p}, v)_{\partial D^{k}}=\oint_{\Gamma}\{\boldsymbol{p}\} \cdot \llbracket v \rrbracket d s+\oint_{\Gamma_{i}}\{v\} \llbracket \boldsymbol{p} \rrbracket d s .
$$

Summing all the terms of 3.6 - 3.8 and application of this Lemma 4.1, we obtain

$$
\begin{aligned}
\int_{\Omega} \boldsymbol{q}_{h} \cdot \nabla v d \boldsymbol{x}-\oint_{\Gamma}\left\{\widehat{\boldsymbol{q}}_{h}\right\} \cdot \llbracket v \rrbracket d s-\oint_{\Gamma_{i}}\{v\} \llbracket \widehat{\boldsymbol{q}}_{h} \rrbracket d s & =\int_{\Omega} f v d \boldsymbol{x} \\
\int_{\Omega} \boldsymbol{q}_{h} \cdot \boldsymbol{\phi} d \boldsymbol{x} & =\int_{\Omega}{ }_{L} I_{\boldsymbol{x}}^{\overline{\boldsymbol{\alpha}}} \boldsymbol{p}_{h} \cdot \boldsymbol{\phi} d \boldsymbol{x} \\
\int_{\Omega} \boldsymbol{p}_{h} \cdot \boldsymbol{\pi} d \boldsymbol{x} & =-\int_{\Omega} u_{h} \nabla \cdot \boldsymbol{\pi} d \boldsymbol{x}+\oint_{\Gamma}\{\boldsymbol{\pi}\} \cdot \llbracket \widehat{u}_{h} \rrbracket d s+\oint_{\Gamma_{i}}\left\{\widehat{u}_{h}\right\} \llbracket \boldsymbol{\pi} \rrbracket d s .(4)
\end{aligned}
$$

Now, we express $\boldsymbol{p}_{h}$ as a function $u_{h}$. To achieve this we use 4.1 and the integration by parts formula

$$
-\int_{\Omega} \nabla \cdot \pi \psi d \boldsymbol{x}=\int_{\Omega} \boldsymbol{\pi} \cdot \nabla \psi d \boldsymbol{x}-\oint_{\Gamma}\{\boldsymbol{\pi}\} \cdot \llbracket \psi \rrbracket d s-\oint_{\Gamma_{i}}\{\psi\} \llbracket \pi \rrbracket d s .
$$

which is valid for all $\psi \in L^{2}\left(\Omega_{h}\right), \boldsymbol{\pi} \in\left(H^{1}\left(\Omega_{h}\right)\right)^{2}$.

Setting $\psi=u_{h}$ in 4.5 and we can rewrite 4.4 as

$$
\int_{\Omega} \boldsymbol{p}_{h} \cdot \boldsymbol{\pi} d \boldsymbol{x}=\int_{\Omega} \boldsymbol{\pi} \cdot \nabla u_{h} d \boldsymbol{x}+\oint_{\Gamma}\{\boldsymbol{\pi}\} \cdot \llbracket \widehat{u}_{h}-u_{h} \rrbracket d s+\oint_{\Gamma_{i}}\left\{\widehat{u}_{h}-u_{h}\right\} \llbracket \boldsymbol{\pi} \rrbracket d s, \quad \forall \boldsymbol{\pi} \in\left(V_{h}\right)^{2} .
$$

Here, the numerical flux is single valued (i.e., $\left\{\widehat{u}_{h}\right\}=\widehat{u}_{h}$ and $\llbracket \widehat{u}_{h} \rrbracket=0$ ), we obtain

$$
\int_{\Omega} \boldsymbol{p}_{h} \cdot \boldsymbol{\pi} d \boldsymbol{x}=\int_{\Omega} \boldsymbol{\pi} \cdot \nabla u_{h} d \boldsymbol{x}-\oint_{\Gamma}\{\boldsymbol{\pi}\} \cdot \llbracket u_{h} \rrbracket d s+\oint_{\Gamma_{i}} \widehat{u}_{h} \llbracket \pi \rrbracket d s-\oint_{\Gamma_{i}}\left\{u_{h}\right\} \llbracket \boldsymbol{\pi} \rrbracket d s .
$$

In Table 1, we can rewrite all numerical fluxes $\widehat{u}_{h}$ as

$$
\widehat{u}_{h}=\left\{u_{h}\right\}+\eta \cdot \llbracket u_{h} \rrbracket \text { on } \Gamma_{i}, \quad \widehat{u}_{h}=0 \text { on } \partial \Omega .
$$

We impose homogeneous boundary conditions on $u_{h}$ along $\Gamma_{b}=\Gamma / \Gamma_{i}$ and substituting in 4.7 , we obtain

$$
\int_{\Omega} \boldsymbol{p}_{h} \cdot \boldsymbol{\pi} d \boldsymbol{x}=\int_{\Omega} \boldsymbol{\pi} \cdot \nabla u_{h} d \boldsymbol{x}-\oint_{\Gamma_{b}} \mathbf{n} \cdot \boldsymbol{\pi} \llbracket u_{h} \rrbracket d s-\oint_{\Gamma_{i}}\{\boldsymbol{\pi}\} \cdot \llbracket u_{h} \rrbracket d s+\eta \oint_{\Gamma_{i}} \llbracket \boldsymbol{\pi} \rrbracket \llbracket u_{h} \rrbracket d s .
$$


We define a lifting operator $L\left(\theta_{h}\right) \in\left(V_{h}\right)^{2}$ for $\theta \in V_{h}$ as

$$
\int_{\Omega} L(\theta) \cdot \boldsymbol{\pi} d \boldsymbol{x}=\oint_{\Gamma_{b}} \mathbf{n} \cdot \boldsymbol{\pi} \theta d s+\oint_{\Gamma_{i}}\{\boldsymbol{\pi}\} \cdot \llbracket \theta \rrbracket d s-\oint_{\Gamma_{i}} \eta \llbracket \pi \rrbracket \llbracket \theta \rrbracket d s,
$$

and obtain from 4.9

$$
\int_{\Omega} \boldsymbol{p}_{h} \cdot \boldsymbol{\pi} d \boldsymbol{x}=\int_{\Omega}\left(\nabla u_{h}-L\left(u_{h}\right)\right) \cdot \boldsymbol{\pi} d \boldsymbol{x}
$$

or

$$
\boldsymbol{p}_{h}=\nabla u_{h}-L\left(u_{h}\right)
$$

which inserted into 4.3 , we obtain

$$
\boldsymbol{q}_{h}={ }_{L} I_{x}^{\bar{\alpha}}\left(\nabla u_{h}-L\left(u_{h}\right)\right) .
$$

Substituting in 4.2 , we obtain the following bilinear form as

$$
\begin{aligned}
B_{h}\left(u_{h}, v\right)= & \int_{\Omega} \nabla v \cdot{ }_{L} I_{\boldsymbol{x}}^{\overline{\boldsymbol{\alpha}}}\left(\nabla u_{h}\right) d \boldsymbol{x}-\int_{\Omega}{ }_{L} I_{\boldsymbol{x}}^{\overline{\boldsymbol{\alpha}}}\left(L\left(u_{h}\right)\right) \cdot \nabla v d \boldsymbol{x} \\
& -\int_{\Omega} L(v) \cdot{ }_{L} I_{x}^{\overline{\boldsymbol{\alpha}}}\left(\nabla u_{h}\right) d \boldsymbol{x}+\int_{\Omega} L(v) \cdot{ }_{L} I_{x}^{\overline{\boldsymbol{\alpha}}}\left(L\left(u_{h}\right)\right) d x+\oint_{\Gamma_{b}} \lambda \llbracket u_{h} \rrbracket \cdot \llbracket v \rrbracket d s+\oint_{\Gamma_{i}} \lambda \llbracket u_{h} \rrbracket \cdot \llbracket v \rrbracket d s \\
= & \int_{\Omega}\left({ }_{L} I_{\boldsymbol{x}}^{\overline{\boldsymbol{\alpha}}}\left(\nabla u_{h}\right)-{ }_{L} I_{\boldsymbol{x}}^{\overline{\boldsymbol{\alpha}}}\left(L\left(u_{h}\right)\right)\right) \cdot(\nabla v-L(v)) d \boldsymbol{x}+\oint_{\Gamma_{i}} \lambda \llbracket u_{h} \rrbracket \cdot \llbracket v \rrbracket d s+\oint_{\Gamma_{b}} \lambda u_{h} v d s,
\end{aligned}
$$

Taking $\boldsymbol{\pi}={ }_{R} I_{x}^{\bar{\alpha}} \phi=\left({ }_{x} \mathcal{I}_{b}^{2-\alpha} \phi^{x},{ }_{y} \mathcal{I}_{d}^{2-\beta} \phi^{y}\right)$ in the identity 4.7 we may then rewrite 4.3 as follows:

$$
\int_{\Omega} \boldsymbol{q}_{h} \cdot \boldsymbol{\phi} d \boldsymbol{x}=\int_{\Omega}{ }_{R} I_{x}^{\bar{\alpha}} \boldsymbol{\phi} \cdot \nabla u_{h} d \boldsymbol{x}-\oint_{\Gamma}\left\{{ }_{R} I_{x}^{\bar{\alpha}} \boldsymbol{\phi}\right\} \cdot \llbracket u_{h} \rrbracket d s+\oint_{\Gamma_{i}} \widehat{u}_{h} \llbracket{ }_{R} I_{x}^{\bar{\alpha}} \phi \rrbracket d s-\oint_{\Gamma_{i}}\left\{u_{h}\right\} \llbracket{ }_{R} I_{x}^{\bar{\alpha}} \boldsymbol{\phi} \rrbracket d s,
$$

Taking $\phi=\nabla v$ and substituting in 4.2 , we obtain

$$
B_{h}\left(u_{h}, v\right)=\int_{\Omega} f v d \boldsymbol{x}
$$

where

$$
\begin{aligned}
B_{h}\left(u_{h}, v\right)= & \int_{\Omega}{ }_{R} I_{x}^{\overline{\boldsymbol{\alpha}}}(\nabla v) \cdot \nabla u_{h} d \boldsymbol{x}-\oint_{\Gamma}\left\{{ }_{R} I_{\boldsymbol{x}}^{\overline{\boldsymbol{\alpha}}} \nabla v\right\} \cdot \llbracket u_{h} \rrbracket d s+\oint_{\Gamma_{i}} \widehat{u}_{h} \llbracket{ }_{R} I_{x}^{\bar{\alpha}} \nabla v \rrbracket d s \\
& -\oint_{\Gamma_{i}}\left\{u_{h}\right\} \llbracket{ }_{R} I_{x}^{\bar{\alpha}} \nabla v \rrbracket d s-\oint_{\Gamma}\left\{\widehat{\boldsymbol{q}}_{h}\right\} \cdot \llbracket v \rrbracket d s-\oint_{\Gamma_{i}}\{v\} \llbracket \widehat{\boldsymbol{q}}_{h} \rrbracket d s,
\end{aligned}
$$

From 4.5 with $\pi={ }_{L} I_{\boldsymbol{x}}^{\overline{\boldsymbol{\alpha}}}\left(\nabla u_{h}\right)$ and $\psi=v$, we recover the identity

$$
\int_{\Omega}{ }_{L} I_{x}^{\bar{\alpha}}\left(\nabla u_{h}\right) \cdot \nabla v d \boldsymbol{x}=-\int_{\Omega} \nabla \cdot\left({ }_{L} I_{x}^{\bar{\alpha}}\left(\nabla u_{h}\right)\right) v d \boldsymbol{x}+\oint_{\Gamma}\left\{{ }_{L} I_{x}^{\bar{\alpha}}\left(\nabla u_{h}\right)\right\} \cdot \llbracket v \rrbracket d s+\oint_{\Gamma_{i}}\{v\} \llbracket{ }_{L} I_{x}^{\bar{\alpha}}\left(\nabla u_{h}\right) \rrbracket d s,
$$

which inserted into 4.17) yields

$$
\begin{aligned}
B_{h}\left(u_{h}, v\right)= & -\int_{\Omega} \nabla \cdot{ }_{L} I_{x}^{\bar{\alpha}}\left(\nabla u_{h}\right) v d \boldsymbol{x}+\oint_{\Gamma}\left(\left(\left\{{ }_{L} I_{x}^{\bar{\alpha}}\left(\nabla u_{h}\right)\right\}-\left\{\widehat{\boldsymbol{q}}_{h}\right\}\right) \cdot \llbracket v \rrbracket-\left\{{ }_{R} I_{x}^{\bar{\alpha}} \nabla v\right\} \cdot \llbracket u_{h} \rrbracket\right) d s \\
& +\oint_{\Gamma_{i}}\left(\left(\widehat{u}_{h}-\left\{u_{h}\right\}\right) \llbracket{ }_{R} I_{x}^{\bar{\alpha}} \nabla v \rrbracket+\llbracket{ }_{L} I_{x}^{\bar{\alpha}}\left(\nabla u_{h}\right)-\widehat{\boldsymbol{q}}_{h} \rrbracket\{v\}\right) d s,
\end{aligned}
$$


To test consistency, let $u$ solve the fractional elliptic problem. Then, we obtain the following bilinear form as

$$
\begin{aligned}
B_{h}(u, v)= & -\int_{\Omega} \nabla \cdot{ }_{L} I_{x}^{\bar{\alpha}}(\nabla u) v d \boldsymbol{x}+\oint_{\Gamma}\left(\left(\left\{{ }_{L} I_{x}^{\bar{\alpha}}(\nabla u)\right\}-\{\widehat{\boldsymbol{q}}\}\right) \cdot \llbracket v \rrbracket-\left\{{ }_{R} I_{x}^{\bar{\alpha}} \nabla v\right\} \cdot \llbracket u \rrbracket\right) d s \\
& +\oint_{\Gamma_{i}}\left((\widehat{u}-\{u\}) \llbracket{ }_{R} I_{x}^{\bar{\alpha}} \nabla v \rrbracket+\llbracket{ }_{L} I_{x}^{\bar{\alpha}}(\nabla u)-\widehat{\boldsymbol{q}} \rrbracket\{v\}\right) d s,
\end{aligned}
$$

since $\{u\}=u,\left\{{ }_{L} I_{x}^{\bar{\alpha}}(\nabla u)\right\}={ }_{L} I_{x}^{\bar{\alpha}}(\nabla u), \llbracket u \rrbracket=\llbracket{ }_{L} I_{x}^{\bar{\alpha}}\left(\nabla u_{h}\right) \rrbracket=0$. If we consider the numerical flux is consistent , we obtain

$$
B_{h}(u, v)=-\int_{\Omega} \nabla \cdot{ }_{L} I_{\boldsymbol{x}}^{\overline{\boldsymbol{\alpha}}}(\nabla u) v d \boldsymbol{x}+\oint_{\Gamma}\left(\left({ }_{L} I_{\boldsymbol{x}}^{\overline{\boldsymbol{\alpha}}}(\nabla u)-\left\{\widehat{\boldsymbol{q}}_{h}\right\}\right) \cdot \llbracket v \rrbracket\right) d s-\oint_{\Gamma_{i}} \llbracket \widehat{\boldsymbol{q}} \rrbracket\{v\} d s,
$$

Then 4.13 implies that

$$
\boldsymbol{q}={ }_{L} I_{x}^{\bar{\alpha}}(\nabla u-L(u))={ }_{L} I_{x}^{\bar{\alpha}}(\nabla u),
$$

In Table 1 if we consider all numerical fluxes are consistent, we then get that $\llbracket \widehat{\boldsymbol{q}} \rrbracket=0$ and $\{\widehat{\boldsymbol{q}}\}={ }_{L} I_{\boldsymbol{x}}^{\overline{\boldsymbol{\alpha}}}(\nabla u)$. Inserting these relations in 4.27 we obtain

$$
B_{h}(u, v)=\int_{\Omega} f v d \boldsymbol{x}
$$

Thus the primal formulation 4.23 is consistent only that the numerical fluxes are consistent, for all $v \in V_{h}$. Furthermore by Galerkin orthogonality, we can be written 4.16 as

$$
B_{h}\left(u-u_{h}, \varphi\right)=0, \quad \varphi \in V_{h}
$$

Let $\psi$ solve

$$
-\frac{\partial^{\alpha} \psi}{\partial x^{\alpha}}-\frac{\partial^{\beta} \psi}{\partial y^{\beta}}=g, \quad \psi=0, \quad x \in \partial \Omega
$$

if we assume that the adjoint problem

$$
B_{h}(v, \psi)=\int_{\Omega} v g d \boldsymbol{x}, \quad v \in H_{0}^{2}
$$

In a similar fashion, we obtain that

$$
B_{h}(\varphi, \psi)=\int_{\Omega} g \varphi d \boldsymbol{x}+\oint_{\Gamma} \llbracket \widehat{u}(\varphi) \rrbracket \cdot{ }_{R} I_{x}^{\bar{\alpha}}(\nabla \psi) d s-\oint_{\Gamma_{i}} \llbracket \widehat{\boldsymbol{q}}(\varphi) \rrbracket \psi d s .
$$

If we consider the numerical fluxes are conservative $(\llbracket \widehat{u}(\varphi) \rrbracket=0$ and $\llbracket \widehat{\boldsymbol{q}}(\varphi) \rrbracket=0)$. Thus, the solution to the adjoint problem is consistent.

\section{Boundedness, stability and error estimate}

To carry out error analysis, we first discuss the stability and boundedness of the bilinear form $B_{h}$. 


\subsection{Boundedness and stability}

To propose the stability and boundedness of the primal form $B_{h}$, let's define the energy norm for $v \in V_{h}$

$$
\|\mid v\|\left\|^{2}=\int_{c}^{d}\right\| v_{x}(\cdot, y)\left\|_{H^{\frac{\alpha_{1}}{2}(a, b)}}^{2} d y+\int_{a}^{b}\right\| v_{y}(x, \cdot)\left\|_{H^{\frac{\alpha_{2}}{2}(c, d)}}^{2} d x+\right\| h^{\frac{-1}{2}} \llbracket v \rrbracket\left\|_{\Gamma_{i}}^{2}+\right\| h^{\frac{-1}{2}} v \|_{\Gamma_{b}}^{2},
$$

where we define the boundary norms as

$$
\|v\|_{\Gamma_{i}}^{2}=\oint_{\Gamma_{i}} v^{2} d \boldsymbol{x}, \quad\|v\|_{\Gamma_{b}}^{2}=\oint_{\Gamma_{b}} v^{2} d \boldsymbol{x}
$$

Lemma 5.1. (See [59]) There exists a generic constant $C$ being independent of $h$, for any $v \in V_{h}$, such that

$$
\|v\|_{\partial D} \leq C h^{-1 / 2}\|v\|_{D}
$$

Next we establish the continuity and coercivity of the bilinear form 4.14.

Theorem 5.1. There exist positive constants $C_{k}, C_{s}$ for any $u_{h}, v \in V_{h}$, the primal bilinear form $B_{h}$ that is,

(i) Bounded: $B_{h}\left(u_{h}, v\right) \leq C_{k}\left|\left\|u_{h}|\|\mid\| v\|\|\right.\right.$.

(ii) Coercive: $B_{h}(v, v) \geq C_{s}\|\| v\|\|^{2}$.

Proof. We can be written 4.14 as

$$
B_{h}(u, v)=I+I I+I I I+I V,
$$

where

$$
\begin{aligned}
I & =\int_{\Omega} \nabla v \cdot{ }_{L} I_{x}^{\bar{\alpha}}(\nabla u) d \boldsymbol{x}, \\
I I & =-\int_{\Omega}{ }_{L} I_{\boldsymbol{x}}^{\bar{\alpha}}(\nabla u) \cdot L(v) d \boldsymbol{x}-\int_{\Omega}{ }_{L} I_{\boldsymbol{x}}^{\bar{\alpha}}(L(u)) \cdot \nabla v d \boldsymbol{x}, \\
I I I & =\int_{\Omega}{ }_{L} I_{x}^{\bar{\alpha}}(L(u)) \cdot L(v) d \boldsymbol{x} d \boldsymbol{x}, \\
I V & =\oint_{\Gamma_{i}} \lambda \llbracket u \rrbracket \cdot \llbracket v \rrbracket d s+\oint_{\Gamma_{b}} \lambda u v d s .
\end{aligned}
$$

For the $I$ term, using Cauchy-Schwarz inequality, we obtain

$$
\begin{aligned}
I \leq c_{1} & \left(\int_{c}^{d}\left\|v_{x}(\cdot, y)\right\|_{H^{\frac{\alpha_{1}}{2}(a, b)}}^{2} d y+\int_{a}^{b}\left\|v_{y}(x, \cdot)\right\|_{H^{\frac{\alpha_{2}}{2}(c, d)}}^{2} d x\right)^{\frac{1}{2}}\left(\int_{c}^{d}\left\|u_{x}(\cdot, y)\right\|_{H^{\frac{\alpha_{1}}{2}(a, b)}}^{2} d y\right. \\
& \left.+\int_{a}^{b}\left\|u_{y}(x, \cdot)\right\|_{H^{\frac{\alpha_{2}}{2}(c, d)}}^{2} d x\right)^{\frac{1}{2}} \leq C \mid\|u\|\|\|\|v\| .
\end{aligned}
$$

For the $I I$ term, using Cauchy-Schwarz inequality, we obtain

$$
\begin{aligned}
I I \leq & c_{1}\left(\int_{c}^{d}\left\|u_{x}(\cdot, y)\right\|_{H^{\frac{\alpha_{1}}{2}}(a, b)}^{2} d y+\int_{a}^{b}\left\|u_{y}(x, \cdot)\right\|_{H^{\frac{\alpha_{2}}{2}(c, d)}}^{2} d x\right)^{\frac{1}{2}}\left\|_{R} I_{x}^{\frac{\bar{\alpha}}{2}}(L(v))\right\|_{\Omega} \\
& +c_{2}\left\|_{R} I_{x}^{\frac{\bar{\alpha}}{2}}(L(u))\right\|_{\Omega}\left(\int_{c}^{d}\left\|v_{x}(\cdot, y)\right\|_{H^{\frac{\alpha_{1}}{2}(a, b)}}^{2} d y+\int_{a}^{b}\left\|v_{y}(x, \cdot)\right\|_{H^{\frac{\alpha_{2}}{2}(c, d)}}^{2} d x\right)^{\frac{1}{2}},
\end{aligned}
$$


employing Lemma 2.2 , we get

$$
\begin{aligned}
I I & \leq c_{3}\left(\int_{c}^{d}\left\|u_{x}(\cdot, y)\right\|_{H^{\frac{\alpha_{1}}{2}(a, b)}}^{2} d y+\int_{a}^{b}\left\|u_{y}(x, \cdot)\right\|_{H^{\frac{\alpha_{2}}{2}(c, d)}}^{2} d x\right)^{\frac{1}{2}}\|L(v)\|_{\Omega} \\
& +c_{4}\|L(u)\|_{\Omega}\left(\int_{c}^{d}\left\|v_{x}(\cdot, y)\right\|_{H^{\frac{\alpha_{1}}{2}(a, b)}}^{2} d y+\int_{a}^{b}\left\|v_{y}(x, \cdot)\right\|_{H^{\frac{\alpha_{2}}{2}(c, d)}}^{2} d x\right)^{\frac{1}{2}} .
\end{aligned}
$$

Exploring the inverse inequality, 5.1, one can furthermore show that 50

$$
\|L(u)\|_{\Omega} \leq c\left(\left\|h^{\frac{-1}{2}} \llbracket u \rrbracket\right\|_{\Gamma_{i}}^{2}+\left\|h^{\frac{-1}{2}} u\right\|_{\Gamma_{b}}^{2}\right)^{\frac{1}{2}} .
$$

Hence

$$
\begin{aligned}
I I \leq & c_{3}\left(\int_{c}^{d}\left\|u_{x}(\cdot, y)\right\|_{H^{\frac{\alpha_{1}}{2}(a, b)}}^{2} d y+\int_{a}^{b}\left\|u_{y}(x, \cdot)\right\|_{H^{\frac{\alpha_{2}}{2}(c, d)}}^{2} d x\right)^{\frac{1}{2}}\left(\left\|h^{\frac{-1}{2}} \llbracket v \rrbracket\right\|_{\Gamma_{i}}^{2}+\left\|h^{\frac{-1}{2}} v\right\|_{\Gamma_{b}}^{2}\right)^{\frac{1}{2}} \\
& +c_{4}\left(\left\|h^{\frac{-1}{2}} \llbracket u \rrbracket\right\|_{\Gamma_{i}}^{2}+\left\|h^{\frac{-1}{2}} u\right\|_{\Gamma_{b}}^{2}\right)^{\frac{1}{2}}\left(\int_{c}^{d}\left\|v_{x}(\cdot, y)\right\|_{H^{\frac{\alpha_{1}}{2}(a, b)}}^{2} d y+\int_{a}^{b}\left\|v_{y}(x, \cdot)\right\|_{H^{\frac{\alpha_{2}}{2}(c, d)}}^{2} d x\right)^{\frac{1}{2}} \\
\leq & C\|\| u\|\|\|v\| .
\end{aligned}
$$

For the III term, using Cauchy-Schwarz inequality and employing Lemma 2.2, we obtain

$$
\begin{aligned}
I I I & \leq c_{1}\left\|{ }^{L} I_{x}^{\bar{\alpha}}(L(u))\right\|_{\Omega}\|L(v)\|_{\Omega} \leq c_{2}\|L(u)\|_{\Omega}\|L(v)\|_{\Omega}, \\
& \leq c_{3}\left(\left\|h^{\frac{-1}{2}} \llbracket u \rrbracket\right\|_{\Gamma_{i}}^{2}+\left\|h^{\frac{-1}{2}} u\right\|_{\Gamma_{b}}^{2}\right)^{\frac{1}{2}}\left(\left\|h^{\frac{-1}{2}} \llbracket v \rrbracket\right\|_{\Gamma_{i}}^{2}+\left\|h^{\frac{-1}{2}} v\right\|_{\Gamma_{b}}^{2}\right)^{\frac{1}{2}} \leq C\|\| u\|\|\|\| v\|\|
\end{aligned}
$$

For the $I V$ term, we recall that $h=\min \left(\left(h^{k}\right)^{-},\left(h^{k}\right)^{+}\right)$and assume that the local stabilization factor as $\lambda=\frac{\tilde{\lambda}^{k}}{h}$. With this, we recover

$$
\begin{aligned}
I V & \leq C\left(\left\|\left(\tilde{\lambda}^{k}\right)^{\frac{1}{2}} h^{\frac{-1}{2}} \llbracket u \rrbracket\right\|_{\Gamma_{i}}\left\|\left(\tilde{\lambda}^{k}\right)^{\frac{1}{2}} h^{\frac{-1}{2}} \llbracket v \rrbracket\right\|_{\Gamma_{i}}+\left\|\left(\tilde{\lambda}^{k}\right)^{\frac{1}{2}} h^{\frac{-1}{2}} u\right\|_{\Gamma_{b}}\left\|\left(\tilde{\lambda}^{k}\right)^{\frac{1}{2}} h^{\frac{-1}{2}} v\right\|_{\Gamma_{b}}\right) \\
& \leq C_{k}|\|u|\|\mid\| v\|\|,
\end{aligned}
$$

where $\tilde{\lambda}^{k}$ indicates that the local constant is depending on the local order of approximation.

Combining (5.9), (5.13), 5.14), 5.15), and (5.4), we obtain $B_{h}(u, v) \leq C_{k} \mid\|u\|\|\|\|v\| \|$. We are finished with the continuity.

To obtain the coercivity of the bilinear form 4.14 can be written

$$
\begin{aligned}
B_{h}(v, v)= & \int_{\Omega} \nabla v \cdot{ }_{L} I_{\boldsymbol{x}}^{\bar{\alpha}}(\nabla v) d \boldsymbol{x}+\int_{\Omega} L(v) \cdot{ }_{L} I_{\boldsymbol{x}}^{\bar{\alpha}}(L(v)) d x-\int_{\Omega}{ }_{L} I_{\boldsymbol{x}}^{\bar{\alpha}}(L(v)) \cdot \nabla v d \boldsymbol{x} \\
& -\int_{\Omega} L(v) \cdot{ }_{L} I_{x}^{\bar{\alpha}}(\nabla v) d \boldsymbol{x}+\oint_{\Gamma_{b}} \lambda \llbracket v \rrbracket \cdot \llbracket v \rrbracket d s+\oint_{\Gamma_{i}} \lambda \llbracket v \rrbracket \cdot \llbracket v \rrbracket d s,
\end{aligned}
$$


Employing Young's inequality, we obtain

$$
\begin{aligned}
B_{h}(v, v) \geq & \int_{\Omega} \nabla v \cdot{ }_{L} I_{x}^{\bar{\alpha}}(\nabla v) d \boldsymbol{x}+\int_{\Omega} L(v) \cdot{ }_{L} I_{x}^{\bar{\alpha}}(L(v)) d \boldsymbol{x}-\frac{1}{2 \varepsilon_{1}}\left\|_{R} I_{x}^{\frac{\bar{\alpha}}{2}}(L(v))\right\|_{\Omega}^{2}-\frac{1}{2 \varepsilon_{2}}\left\|_{R} I_{x}^{\frac{\bar{\alpha}}{2}}(L(v))\right\|_{\Omega}^{2} \\
& -\frac{\varepsilon_{1}}{2}\left(\int_{c}^{d}\left\|v_{x}(\cdot, y)\right\|_{H^{\frac{\alpha_{1}}{2}}(a, b)}^{2} d y+\int_{a}^{b}\left\|v_{y}(x, \cdot)\right\|_{H^{\frac{\alpha_{2}}{2}}(c, d)}^{2} d x\right) \\
& -\frac{\varepsilon_{2}}{2}\left(\int_{c}^{d}\left\|v_{x}(\cdot, y)\right\|_{H^{\frac{\alpha_{1}}{2}}(a, b)}^{2} d y+\int_{a}^{b}\left\|v_{y}(x, \cdot)\right\|_{H^{\frac{\alpha_{2}}{2}(c, d)}}^{2} d x\right)+\oint_{\Gamma_{b}} \lambda \llbracket v \rrbracket \cdot \llbracket v \rrbracket d s+\oint_{\Gamma_{i}} \lambda \llbracket v \rrbracket \cdot \llbracket v \rrbracket d s \\
\geq & \int_{\Omega} \nabla v \cdot{ }_{L} I_{x}^{\bar{\alpha}}(\nabla v) d \boldsymbol{x}+\int_{\Omega} L(v) \cdot{ }_{L} I_{x}^{\bar{\alpha}}(L(v)) d \boldsymbol{x}-\frac{1}{2 \varepsilon_{1}}\left\|_{R} I_{x}^{\frac{\bar{\alpha}}{2}}(L(v))\right\|_{\Omega}^{2}-\frac{1}{2 \varepsilon_{2}}\left\|_{R} I_{x}^{\frac{\bar{\alpha}}{2}}(L(v))\right\|_{\Omega}^{2} \\
& -c \varepsilon\left(\int_{c}^{d}\left\|v_{x}(\cdot, y)\right\|_{H^{\frac{\alpha_{1}}{2}}(a, b)}^{2} d y+\int_{a}^{b}\left\|v_{y}(x, \cdot)\right\|_{H^{\frac{\alpha_{2}}{2}}(c, d)}^{2} d x\right) \\
& +\oint_{\Gamma_{b}} \lambda \llbracket v \rrbracket \cdot \llbracket v \rrbracket d s+\oint_{\Gamma_{i}} \lambda \llbracket v \rrbracket \cdot \llbracket v \rrbracket d s \\
\geq & \left(\cos \left(\left(\alpha_{1} / 2\right) \pi\right)-c \varepsilon\right) \int_{c}^{d}\left\|v_{x}(\cdot, y)\right\|_{H^{\frac{\alpha}{2}}}^{2}(a, b) \\
& +\int_{\Omega} L(v) \cdot{ }_{L} I_{x}^{\bar{\alpha}}(L(v)) d \boldsymbol{x}-\frac{c}{\varepsilon}\|(L(v))\|_{\Omega}^{2}+\oint_{\Gamma_{b}} \lambda \llbracket v \rrbracket \cdot \llbracket v \rrbracket d s+\oint_{\Gamma_{i}} \lambda \llbracket v \rrbracket \cdot \llbracket v \rrbracket d s,
\end{aligned}
$$

provided $\varepsilon$ is sufficiently small such that $\cos \left(\left(\alpha_{1} / 2\right) \pi\right)>c \varepsilon$ and $\cos \left(\left(\alpha_{2} / 2\right) \pi\right)>c \varepsilon$.

Comparing (5.12) and (5.1) and assume that $\varepsilon<1$, it is clear that

$$
\|L(v)\|_{\Omega}^{2} \leq C_{l}^{2}\|\| v \|^{2}
$$

and

$$
\int_{\Omega} L(v) \cdot{ }_{L} I_{\boldsymbol{x}}^{\overline{\boldsymbol{\alpha}}}(L(v)) d \boldsymbol{x} \leq c C_{l}^{2} \mid\|v\|\left\|^{2} \Longrightarrow \int_{\Omega} L(v) \cdot{ }_{L} I_{\boldsymbol{x}}^{\overline{\boldsymbol{\alpha}}}(L(v)) d \boldsymbol{x} \geq\left(1-\frac{1}{\varepsilon}\right) c C_{l}^{2}\right\|\|v\| \|^{2} .
$$

Combining these pieces, we recover

$$
\begin{aligned}
B_{h}(v, v) & \geq\left(\cos \left(\left(\alpha_{1} / 2\right) \pi\right)-c \varepsilon+c C_{l}^{2}\left(1-\frac{1}{\varepsilon}\right)\right) \int_{c}^{d}\left\|v_{x}(\cdot, y)\right\|_{H^{\frac{\alpha_{1}}{2}}(a, b)}^{2} d y \\
& +\left(\cos \left(\left(\alpha_{2} / 2\right) \pi\right)-c \varepsilon+c C_{l}^{2}\left(1-\frac{1}{\varepsilon}\right)\right) \int_{a}^{b}\left\|v_{y}(x, \cdot)\right\|_{H^{\frac{\alpha_{2}}{2}(c, d)}}^{2} d x \\
& +\left(c C_{l}^{2}\left(1-\frac{1}{\varepsilon}\right)+\tilde{\lambda}\right)\left(\left\|h^{\frac{-1}{2}} \llbracket v \rrbracket\right\|_{\Gamma_{i}}^{2}+\left\|h^{\frac{-1}{2}} v\right\|_{\Gamma_{b}}^{2}\right),
\end{aligned}
$$

where $\tilde{\lambda} \leq \min \left(\tilde{\lambda}^{k}\right)$, with $\tilde{\lambda}^{k}$ being the local stabilization factor on element $k$.

To establish coercivity, we must show that the two terms in $B_{h}(v, v)$ are both positive, provided

$$
\frac{c C_{l}^{2}}{\tilde{\lambda}+c C_{l}^{2}} \leq \varepsilon \leq 1
$$

Hence $B_{h}$ is stability when $\tilde{\lambda}>0$. 


\subsection{Error estimates.}

In order to carry out the error estimates for the DG methods by using the boundedness, consistency and stability properties. We first review the following lemma for our analysis

Theorem 5.2. (See [60]) Assume that $u \in H^{r}\left(D^{k}\right), r>1 / 2$, and that $u_{h}$ represents a piecewise polynomial interpolation of order $N$. Then

$$
\left\|u-u_{h}\right\|_{\Omega, s, h} \leq C \frac{h^{\sigma-s}}{N^{r-2 s-1 / 2}}|u|_{\Omega, \sigma, h},
$$

for $0 \leq s \leq \sigma$, and $\sigma=\min (N+1, r)$.

Theorem 5.3. Let $u \in H^{p}(\Omega)$ and that $u_{h}$ represents a piecewise polynomial of order $N$. Then

$$
|| u-u_{h}|\|| \leq C h^{N}|u|_{\Omega, \sigma, h}
$$

and The $L^{2}$ error

$$
\left\|u-u_{h}\right\|_{\Omega, h} \leq C h^{N+1}|u|_{\Omega, \sigma, h} .
$$

The constant $C$ depends on $N, \alpha, \beta$ and $p$ but not on $h$.

Proof. From Young's theorem [61, Theorem 5.3 and Lemma 5.1, we can rewrite [5.1) as

$$
\begin{aligned}
\|\| u-u_{h} \mid \|^{2}= & \int_{c}^{d}\left\|_{a} \mathcal{I}_{x}^{\frac{\alpha_{1}}{2}} \partial_{x}\left(u(\cdot, y)-u_{h}(\cdot, y)\right)\right\|_{L^{2}(a, b)}^{2} d y+\int_{a}^{b}\left\|_{c} \mathcal{I}_{y}^{\frac{\alpha_{2}}{2}} \partial_{y}\left(u(x, \cdot)-u_{h}(x, \cdot)\right)\right\|_{L^{2}(c, d)}^{2} d x \\
& +\left\|h^{\frac{-1}{2}} \llbracket u-u_{h} \rrbracket\right\|_{\Gamma_{i}}^{2}+\left\|h^{\frac{-1}{2}}\left(u-u_{h}\right)\right\|_{\Gamma_{b}}^{2} \\
\leq & \left\|\frac{1}{\Gamma\left(\frac{\alpha_{1}}{2}\right)} \int_{-a}^{x}(x-z)^{\frac{\alpha_{1}}{2}-1} d z\right\|_{L^{1}(a, b)}^{2} \int_{c}^{d}\left\|\partial_{x}\left(u(\cdot, y)-u_{h}(\cdot, y)\right)\right\|_{L^{2}(a, b)}^{2} d y \\
& \quad+\left\|\frac{1}{\Gamma\left(\frac{\alpha_{2}}{2}\right)} \int_{-c}^{y}(y-z)^{\frac{\alpha_{2}}{2}-1} d z\right\|_{L^{1}(c, d)}^{2} \int_{a}^{b}\left\|\partial_{y}\left(u(x, \cdot)-u_{h}(x, \cdot)\right)\right\|_{L^{2}(c, d)}^{2} d x \\
& \quad+h^{-2}\left\|u-u_{h}\right\|_{\Omega, h}^{2} \\
\leq & C\left\|u-u_{h}\right\|_{\Omega, 1, h}^{2}+h^{-2}\left\|u-u_{h}\right\|_{\Omega, h}^{2} \\
\leq & C(N, s, r) h^{\sigma-1}|u|_{\Omega, \sigma, h} .
\end{aligned}
$$

We rewrite the coercivity result as

$$
B_{h}\left(u_{h}, u_{h}\right) \geq c\left|\left\|u_{h} \mid\right\|^{2}\right.
$$

We define the projection of the exact solution, $\mathcal{P} u$, and the numerical solution, $u_{h}$, and consider

$$
B_{h}\left(u_{h}-\mathcal{P} u, u_{h}-\mathcal{P} u\right)=B_{h}\left(u-\mathcal{P} u, u_{h}-\mathcal{P} u\right) \geq c \mid\left\|u_{h}-\mathcal{P} u\right\| \|^{2}
$$


Using the continuity of $B_{h}$, we can rewrite 5.27 as

$$
\begin{aligned}
C\left|\| u_{h}-\mathcal{P} u\right|||^{2} & \leq B_{h}\left(u-\mathcal{P} u, u_{h}-\mathcal{P} u\right) \\
& \leq c\left|\| u - \mathcal { P } u \| \left\|\left|\left\|u_{h}-\mathcal{P} u\right\|\right| .\right.\right.
\end{aligned}
$$

From (5.25), we obtain

$$
\left\|\left|u_{h}-\mathcal{P} u\right|\right\| \leq C h^{\sigma-1}|u|_{\Omega, \sigma, h}
$$

Employing the triangle inequality, we get

$$
||\left|u-u_{h}\right| \|\left.\left|\leq C h^{\sigma-1}\right| u\right|_{\Omega, \sigma, h} .
$$

Hence the optimal order under energy norm of convergence $\mathcal{O}\left(h^{N}\right)$ for sufficiently smooth solutions.

To obtain optimal order $L^{2}$-error estimates, we consider the auxiliary function $\theta$ as the solution of the adjoint problem

$$
-\frac{\partial^{\alpha} \theta}{\partial x^{\alpha}}-\frac{\partial^{\beta} \theta}{\partial y^{\beta}}=u-u_{h}, \quad \theta=0, \quad x \in \partial \Omega
$$

and we consider the adjoint consistency condition holds

$$
B_{h}(\phi, \theta)=\left(u-u_{h}, \phi\right)_{\Omega}, \quad \forall \phi \in H_{0}^{2}
$$

Taking $\phi=u-u_{h}$ in 5.32 and consider $\theta_{I}$ to be a piecewise linear interpolant of $\theta$, the consistency condition 4.24 continuity of $B_{h}$ and Galerkin orthogonality, we get

$$
\left\|u-u_{h}\right\|_{\Omega, h}^{2}=B_{h}\left(u-u_{h}, \theta\right)=B_{h}\left(u-u_{h}, \theta-\theta_{I}\right) \leq\left\|\mid u-u_{h}\right\|\|\| \theta-\theta_{I} \|_{\Omega, h} .
$$

From elliptic regularity, we obtain

$$
\left\|u-u_{h}\right\|_{\Omega, h}^{2} \leq C h\left|\left\|u-u_{h}||\left|\|\psi\|_{\Omega, 2, h} \leq C h\right|\right\| u-u_{h}\right|||\left\|u-u_{h}\right\|_{\Omega, h} .
$$

Hence, we get the optimal estimate

$$
\left\|u-u_{h}\right\|_{\Omega, h} \leq C h^{\sigma}|u|_{\Omega, \sigma, h} .
$$

This confirms the optimal error estimate of convergence under $L^{2}$ norm is $\mathcal{O}\left(h^{N+1}\right)$ for sufficiently smooth solutions.

\section{Numerical examples}

In this section, we will provide some numerical examples to validate analysis in structured uniform, unstructured and L-shaped domain (see Figures 4 and 5 ). 
Example 6.1. We consider fractional Poisson problem

$$
{ }_{-{ }_{-1}} \mathcal{D}_{x}^{\alpha} u(x, y){ }_{-{ }_{-1}} \mathcal{D}_{y}^{\beta} u(x, y)=f(x, y), \Omega=(-1,1) \times(-1,1)
$$

where

$$
f(x, y)=-\left(y^{2}-1\right)_{-1}^{3} \mathcal{I}_{x}^{2-\alpha}\left(6\left(x^{2}-1\right)\left(5 x^{2}-1\right)\right)-\left(x^{2}-1\right)_{-1}^{3} \mathcal{I}_{y}^{2-\beta}\left(6\left(y^{2}-1\right)\left(5 y^{2}-1\right)\right)
$$

The exact solution is $u(x, y)=\left(x^{2}-1\right)^{3}\left(y^{2}-1\right)^{3}$.

The convergence rates and the numerical $L^{2}$ error of the DG methods of the different formulations on structured uniform meshes are shown in Figures 1 3, confirming optimal $\mathcal{O}\left(h^{N+1}\right)$ order of convergence across. We also compute the condition number of the LDG of discretized matrix $\mathcal{K}_{L D G}$, the central of discretized matrix $\mathcal{K}_{C}$ and the IP of discretized matrix $\mathcal{K}_{I P}$ in Table 2 We shows that the IP and the central methods have almost identical condition number. The choice of the LDG flux leads to a much sparser operator in all cases. From Table 2 it is obvious that the IP method appears to offer a suitable compromise between LDG and central methods. Moreover, we show that the convergence rate $\mathcal{O}\left(h^{N+1}\right)$, which clear that the LDG, IP and central fluxes are optimal in two dimension on unstructured mesh in Tables 3 5

Figure 1: The rate of convergence for the solving the fractional elliptic problem with a stabilized central flux when $\alpha=\beta=1.4$ and $\alpha=\beta=1.99$ on structured uniform mesh for Example 6.1
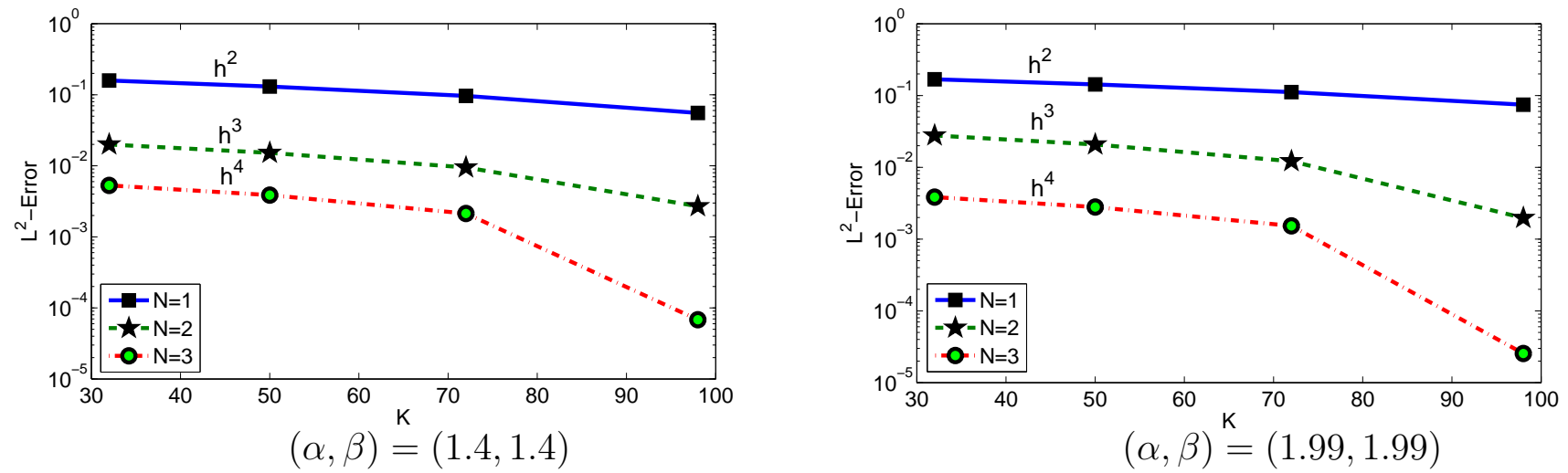
Figure 2: The rate of convergence for the solving the fractional elliptic problem with a stabilized IP flux when $\alpha=\beta=1.4$ and $\alpha=\beta=1.99$ on structured uniform meshes for Example 6.1
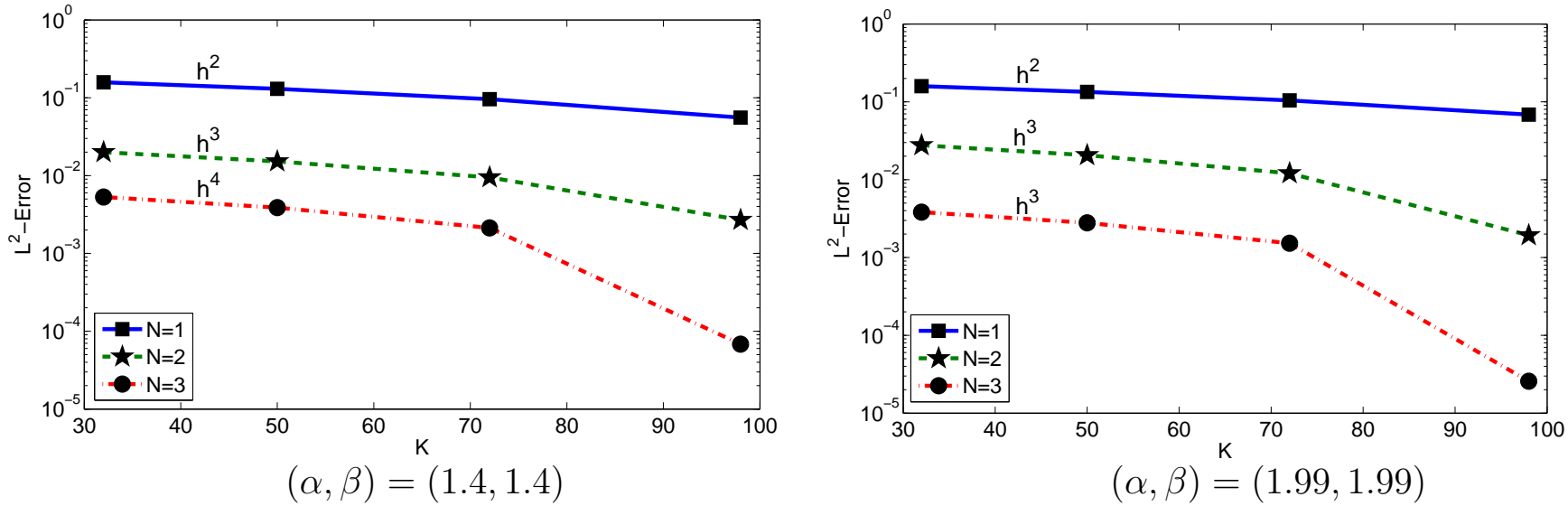

Figure 3: The rate of convergence for the solving the fractional elliptic problem with a stabilized LDG flux when $\alpha=\beta=1.4$ and $\alpha=\beta=1.99$ on structured uniform meshes for Example 6.1
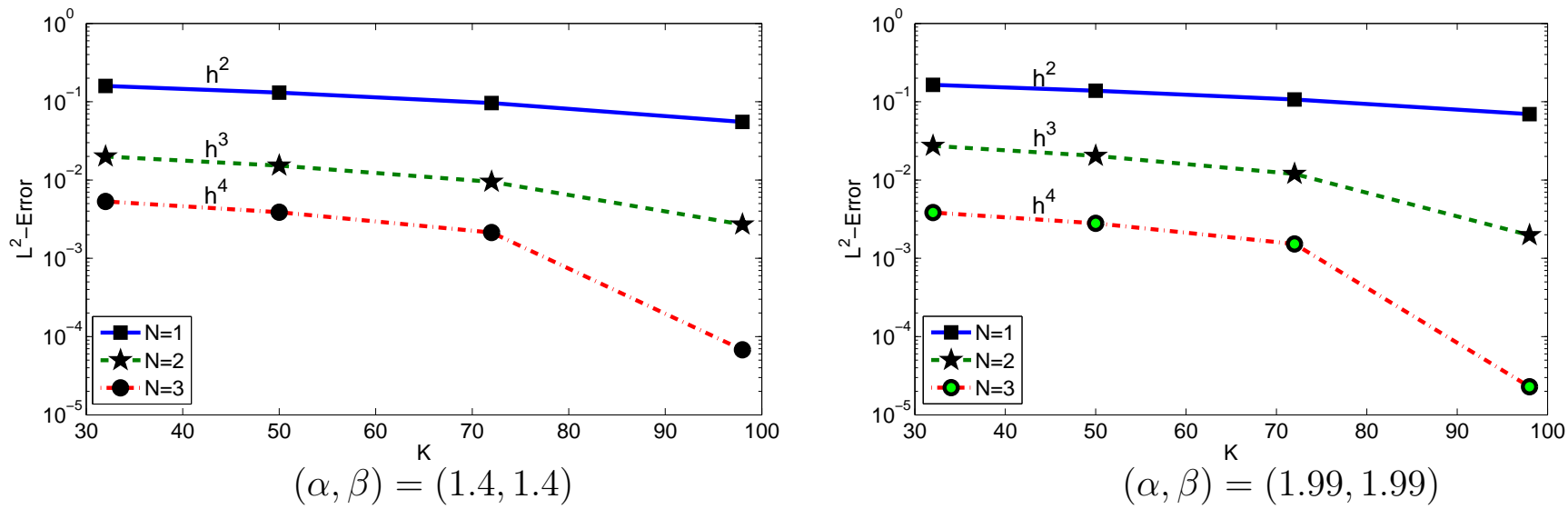
Table 2: The condition number of the matrices for the model homogeneous fractional Poisson problem on structured uniform meshes for Example 6.1

\begin{tabular}{|c|c|c|c|c|c|c|c|}
\hline$(\alpha, \beta)$ & \multicolumn{3}{|c|}{$(1.1,1.1)$} & \multicolumn{4}{|c|}{$(1.99,1.1)$} \\
\hline$K$ & $\mathcal{K}_{C}$ & $\mathcal{K}_{I P}$ & $\mathcal{K}_{L D G}$ & $K$ & $\mathcal{K}_{C}$ & $\mathcal{K}_{I P}$ & $\mathcal{K}_{L D G}$ \\
\hline 8 & 169.25 & 172.23 & 187.25 & 8 & 72.53 & 58.21 & 95.94 \\
\hline 18 & 450.16 & 465.36 & 494.46 & 18 & 175.04 & 122.92 & 228.07 \\
\hline 32 & 912.43 & 963.43 & $1.02 \mathrm{e}+003$ & 32 & 303.56 & 232.63 & 396.87 \\
\hline 50 & $1.52 \mathrm{e}+003$ & $1.62 \mathrm{e}+03$ & $1.71 \mathrm{e}+003$ & 50 & 474.67 & 365.20 & 620.65 \\
\hline$(\alpha, \beta)$ & \multicolumn{3}{|c|}{$(1.6,1.6)$} & \multicolumn{4}{|c|}{$(1.1,1.6)$} \\
\hline$K$ & $\mathcal{K}_{C}$ & $\mathcal{K}_{I P}$ & $\mathcal{K}_{L D G}$ & K & $\mathcal{K}_{C}$ & $\mathcal{K}_{I P}$ & $\mathcal{K}_{L D G}$ \\
\hline 8 & 61.83 & 47.41 & 70.61 & 8 & 90.95 & 84.35 & 106.49 \\
\hline 18 & 124.77 & 103.16 & 150.14 & 18 & 212.31 & 196.86 & 248.01 \\
\hline 32 & 191.42 & 161.73 & 229.85 & 32 & 365.0 & 344.24 & 433.62 \\
\hline 50 & 261.90 & 226.25 & 301.43 & 50 & 545.58 & 530.91 & 638.73 \\
\hline$(\alpha, \beta)$ & \multicolumn{3}{|c|}{$(1.99,1.99)$} & \multicolumn{4}{|c|}{$(1.6,1.99)$} \\
\hline$K$ & $\mathcal{K}_{C}$ & $\mathcal{K}_{I P}$ & $\mathcal{K}_{L D G}$ & $\mathrm{~K}$ & $\mathcal{K}_{C}$ & $\mathcal{K}_{I P}$ & $\mathcal{K}_{L D G}$ \\
\hline 8 & 46.91 & 29.44 & 64.9 & 8 & 55.98 & 40.01 & 73.39 \\
\hline 18 & 81.98 & 47.57 & 112.88 & 18 & 108.5 & 75.76 & 140.36 \\
\hline 32 & 158.91 & 92.45 & 217.56 & 32 & 183.64 & 127.10 & 236.04 \\
\hline 50 & 229.44 & 131.95 & 311.39 & 50 & 261.07 & 182.06 & 336.36 \\
\hline
\end{tabular}



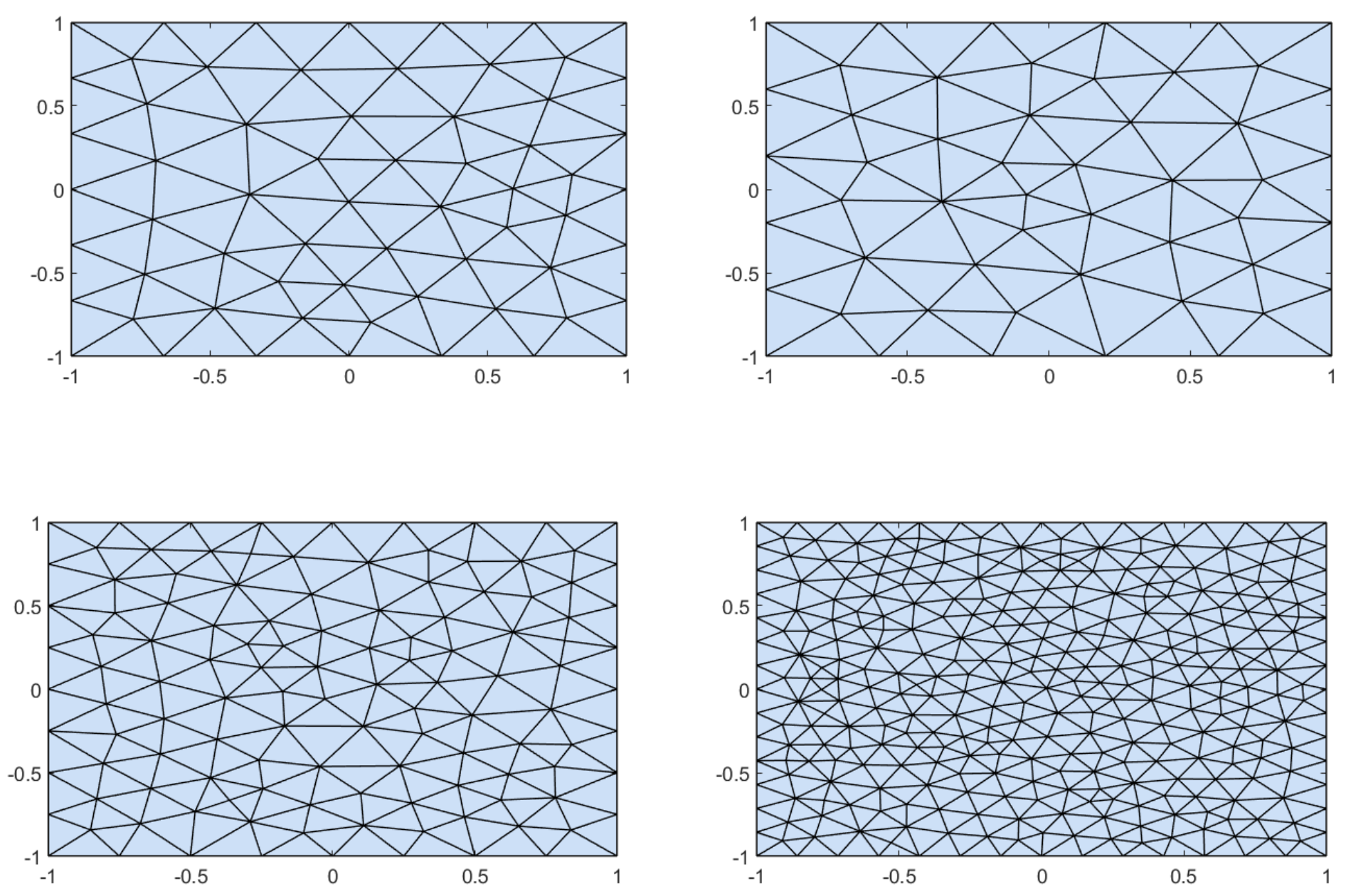

Figure 4: Some unconstructed meshes used in Example 6.1 
Table 3: The convergence order and numerical errors $\left(L^{2}\right)$ for the solving the homogeneous fractional Poisson problem on unstructured meshes for Example 6.1 with a stabilized central flux when $\lambda=1$.

\begin{tabular}{|c|c|c|c|c|c|c|c|}
\hline \multirow{3}{*}{$\begin{array}{c}\mathrm{K} \\
(\alpha, \beta)\end{array}$} & \multicolumn{7}{|c|}{$N=1$} \\
\hline & \multirow{2}{*}{$\begin{array}{c}100 \\
L^{2} \text { error }\end{array}$} & \multicolumn{2}{|l|}{208} & \multicolumn{2}{|c|}{598} & \multicolumn{2}{|l|}{816} \\
\hline & & $L^{2}$ error & order & $L^{2}$ error & order & $L^{2}$ error & order \\
\hline$(1.1,1.1)$ & $5.20 \mathrm{e}-02$ & $2.64 \mathrm{e}-02$ & 2.02 & $8.8 \mathrm{e}-03$ & 2.15 & $6.7 \mathrm{e}-03$ & 1.91 \\
\hline$(1.4,1.4)$ & $5.03 \mathrm{e}-02$ & $2.40 \mathrm{e}-02$ & 2.2 & $8.2 \mathrm{e}-03$ & 2.1 & $6.11 \mathrm{e}-03$ & 2.07 \\
\hline$(1.6,1.6)$ & $5.20 \mathrm{e}-02$ & $2.42 \mathrm{e}-02$ & 2.27 & $8.42 \mathrm{e}-03$ & 2.07 & $6.31 \mathrm{e}-03$ & 2.02 \\
\hline \multirow[t]{2}{*}{$(1.9,1.9)$} & $5.77 \mathrm{e}-02$ & $2.73 \mathrm{e}-02$ & 2.22 & $9.21 \mathrm{e}-03$ & 1.78 & $6.85 \mathrm{e}-03$ & 2.07 \\
\hline & \multicolumn{7}{|c|}{$N=2$} \\
\hline K & 100 & \multicolumn{2}{|l|}{208} & \multicolumn{2}{|c|}{598} & \multicolumn{2}{|l|}{816} \\
\hline$\alpha$ & $L^{2}$ error & $L^{2}$ error & order & $L^{2}$ error & order & $L^{2}$ error & order \\
\hline$(1.1,1.1)$ & $3.79 \mathrm{e}-03$ & $1.55 \mathrm{e}-03$ & 2.66 & $3.58 \mathrm{e}-04$ & 2.87 & $2.359 \mathrm{e}-04$ & 2.92 \\
\hline$(1.4,1.4)$ & $3.01 \mathrm{e}-03$ & $1.07 \mathrm{e}-03$ & 3.07 & $2.21 \mathrm{e}-04$ & 3.09 & $1.42 \mathrm{e}-04$ & 3.09 \\
\hline$(1.6,1.6)$ & $2.87 \mathrm{e}-03$ & $1.028 \mathrm{e}-03$ & 3.05 & $2.11 \mathrm{e}-04$ & 3.1 & $1.35 \mathrm{e}-04$ & 3.12 \\
\hline \multirow[t]{2}{*}{$(1.9,1.9)$} & $2.98 \mathrm{e}-03$ & $1.0724 \mathrm{e}-03$ & 3.04 & $2.27 \mathrm{e}-04$ & 3.04 & $1.44 \mathrm{e}-04$ & 3.18 \\
\hline & \multicolumn{7}{|c|}{$N=3$} \\
\hline K & 130 & 232 & & 324 & & 502 & \\
\hline$\alpha$ & $L^{2}$ error & $L^{2}$ error & order & $L^{2}$ error & order & $L^{2}$ error & order \\
\hline$(1.1,1.1)$ & $3.33 \mathrm{e}-04$ & $1.38 \mathrm{e}-04$ & 3.95 & $6.54 \mathrm{e}-05$ & 4.1 & $2.59 \mathrm{e}-05$ & 4.15 \\
\hline$(1.4,1.4)$ & 2.31e-04 & $8.66 \mathrm{e}-05$ & 4.4 & $4.08 \mathrm{e}-05$ & 4.13 & $1.61 \mathrm{e}-05$ & 4.17 \\
\hline$(1.6,1.6)$ & $2.15 \mathrm{e}-04$ & $8.36 \mathrm{e}-05$ & 4.23 & $3.88 \mathrm{e}-05$ & 4.21 & $1.54 \mathrm{e}-05$ & 4.14 \\
\hline$(1.9,1.9)$ & $1.99 \mathrm{e}-04$ & $7.26 \mathrm{e}-05$ & 4.52 & $3.45 \mathrm{e}-05$ & 4.08 & $1.34 \mathrm{e}-05$ & 4.29 \\
\hline
\end{tabular}


Table 4: The convergence order and numerical errors $\left(L^{2}\right)$ for the solving the homogeneous fractional Poisson problem on unstructured meshes for Example 6.1 with IP flux when $\lambda=\mathcal{O}(h)$.

\begin{tabular}{|c|c|c|c|c|c|c|c|}
\hline & \multicolumn{7}{|c|}{$N=1$} \\
\hline \multirow{2}{*}{$\begin{array}{c}\mathrm{K} \\
(\alpha, \beta)\end{array}$} & 100 & \multicolumn{2}{|l|}{208} & \multicolumn{2}{|l|}{598} & \multicolumn{2}{|c|}{816} \\
\hline & $L^{2}$ error & $L^{2}$ error & order & $L^{2}$ error & order & $L^{2}$ error & order \\
\hline$(1.1,1.1)$ & $5.161 \mathrm{e}-02$ & $2.70 \mathrm{e}-02$ & 1.93 & $9.24 \mathrm{e}-03$ & 2.1 & $6.81 \mathrm{e}-03$ & 2.13 \\
\hline$(1.4,1.4)$ & $5.27 \mathrm{e}-02$ & $2.56 \mathrm{e}-02$ & 2.15 & $9.12 \mathrm{e}-03$ & 2.02 & $6.74 \mathrm{e}-03$ & 2.11 \\
\hline$(1.6,1.6)$ & $5.84 \mathrm{e}-02$ & $2.77 \mathrm{e}-02$ & 2.22 & $9.24 \mathrm{e}-03$ & 2.15 & $6.85 \mathrm{e}-03$ & 2.09 \\
\hline \multirow[t]{2}{*}{$(1.9,1.9)$} & $6.93 \mathrm{e}-02$ & $3.35 \mathrm{e}-02$ & 2.16 & $1.15 \mathrm{e}-02$ & 2.09 & $8.95 \mathrm{e}-03$ & 2.08 \\
\hline & \multicolumn{7}{|c|}{$N=2$} \\
\hline K & 100 & \multicolumn{2}{|l|}{208} & \multicolumn{2}{|c|}{598} & \multicolumn{2}{|l|}{816} \\
\hline$\alpha$ & $L^{2}$ error & $L^{2}$ error & order & $L^{2}$ error & order & $L^{2}$ error & order \\
\hline$(1.1,1.1)$ & $5.18 \mathrm{e}-03$ & $2.24 \mathrm{e}-03$ & 2.49 & $4.87 \mathrm{e}-04$ & 2.98 & $3.11 \mathrm{e}-04$ & 3.13 \\
\hline$(1.4,1.4)$ & $4.42 \mathrm{e}-03$ & $1.9 \mathrm{e}-03$ & 2.51 & $4.24 \mathrm{e}-04$ & 2.94 & $2.7 \mathrm{e}-04$ & 3.15 \\
\hline$(1.6,1.6)$ & $4.26 \mathrm{e}-03$ & $1.80 \mathrm{e}-03$ & 2.56 & $3.89 \mathrm{e}-04$ & 3.0 & $2.53 \mathrm{e}-04$ & 3.01 \\
\hline \multirow[t]{2}{*}{$(1.9,1.9)$} & $4.17 \mathrm{e}-03$ & $1.55 \mathrm{e}-03$ & 2.94 & $3.21 \mathrm{e}-04$ & 3.08 & $2.05 \mathrm{e}-04$ & 3.13 \\
\hline & \multicolumn{7}{|c|}{$N=3$} \\
\hline $\mathrm{K}$ & 130 & 232 & & & & 502 & \\
\hline$\alpha$ & $L^{2}$ error & $L^{2}$ error & order & $L^{2}$ error & order & $L^{2}$ error & order \\
\hline$(1.1,1.1)$ & $5.6 \mathrm{e}-04$ & $1.38 \mathrm{e}-04$ & 3.95 & $6.54 \mathrm{e}-05$ & 4.1 & $2.59 \mathrm{e}-05$ & 4.15 \\
\hline$(1.4,1.4)$ & $3.74 \mathrm{e}-04$ & $8.66 \mathrm{e}-05$ & 4.4 & $4.08 \mathrm{e}-05$ & 4.13 & $1.61 \mathrm{e}-05$ & 4.17 \\
\hline$(1.6,1.6)$ & $3.14 \mathrm{e}-04$ & $8.36 \mathrm{e}-05$ & 4.23 & $3.88 \mathrm{e}-05$ & 4.21 & $1.54 \mathrm{e}-05$ & 4.14 \\
\hline$(1.9,1.9)$ & $2.71 \mathrm{e}-04$ & $1.01 \mathrm{e}-04$ & 4.42 & $3.45 \mathrm{e}-05$ & 4.08 & $1.34 \mathrm{e}-05$ & 4.29 \\
\hline
\end{tabular}


Table 5: The convergence order and numerical errors $\left(L^{2}\right)$ for the solving the homogeneous fractional Poisson problem on unstructured meshes for Example 6.1 with LDG flux when $\lambda=\mathcal{O}(h), \eta= \pm \mathbf{n}$.

\begin{tabular}{|c||ccccccc|}
\hline \hline \multicolumn{1}{|c||}{} & \multicolumn{7}{c|}{$N=1$} \\
\hline $\mathrm{K}$ & 100 & 208 & \multicolumn{2}{c|}{598} & 816 \\
$(\alpha, \beta)$ & $L^{2}$ error & $L^{2}$ error & order & $L^{2}$ error & order & $L^{2}$ error & order \\
\hline$(1.1,1.1)$ & $5.22 \mathrm{e}-02$ & $2.72 \mathrm{e}-02$ & 1.94 & $9.27 \mathrm{e}-03$ & 2.11 & $6.85 \mathrm{e}-03$ & 2.01 \\
$(1.4,1.4)$ & $5.31 \mathrm{e}-02$ & $2.59 \mathrm{e}-02$ & 2.13 & $8.85 \mathrm{e}-03$ & 2.1 & $6.53 \mathrm{e}-03$ & 2.11 \\
$(1.6,1.6)$ & $5.79 \mathrm{e}-02$ & $2.77 \mathrm{e}-02$ & 2.19 & $9.49 \mathrm{e}-03$ & 2.1 & $6.63 \mathrm{e}-03$ & 2.02 \\
$(1.9,1.9)$ & $6.77 \mathrm{e}-02$ & $3.32 \mathrm{e}-02$ & 2.16 & $1.12 \mathrm{e}-02$ & 2.13 & $8.21 \mathrm{e}-03$ & 2.17 \\
\hline \hline
\end{tabular}

Example 6.2. Let us finally simulate the fractional Poisson problem 1.1 over the L-shaped domain $\Omega$ shown in Figure 5 with the forcing term $f(x, y)$ is of the form

$$
f(x, y)=-\left(y^{2}-1\right)_{-1}^{3} \mathcal{I}_{x}^{2-\alpha}\left(6\left(x^{2}-1\right)\left(5 x^{2}-1\right)\right)-\left(x^{2}-1\right)_{-1}^{3} \mathcal{I}_{y}^{2-\beta}\left(6\left(y^{2}-1\right)\left(5 y^{2}-1\right)\right) .
$$

In this case, the exact solution will be $u(x, y)=x^{2}\left(x^{2}-1\right) y^{2}\left(y^{2}-1\right)$.

The computed $L^{2}$ error is shown in Table 6 for the different values of $N, K$ and $\alpha, \beta$ with a stabilized central flux. We note that the convergence of the scheme is performed very well in the L-shaped domain. 

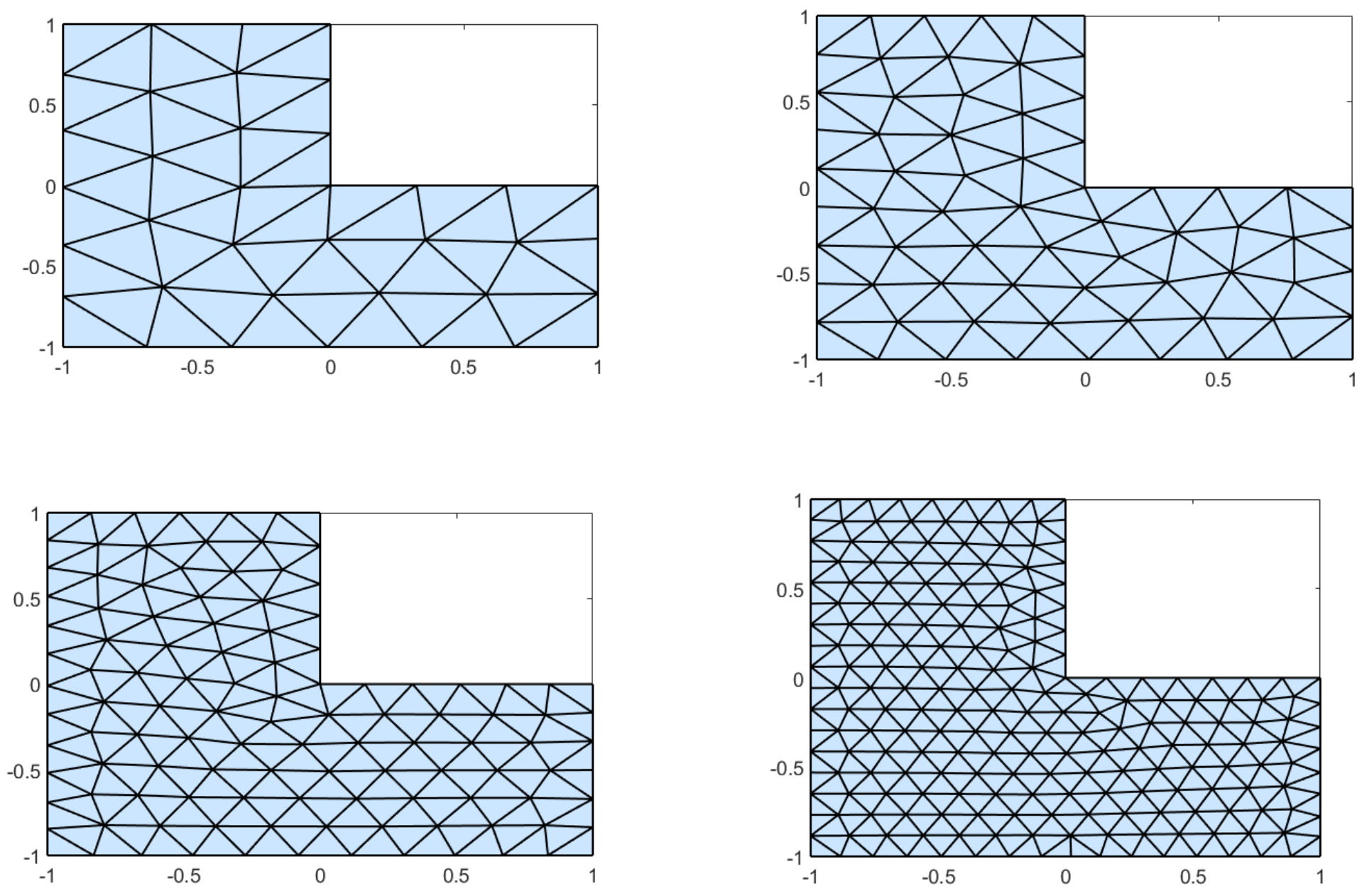

Figure 5: $\quad$ Unconstructed meshes on L-shaped domains used in Example 6.2

Table 6: Numerical errors $\left(L^{2}\right)$ of the the homogeneous fractional Poisson problem on the L-shaped domain.

\begin{tabular}{||c||c||cccc||}
\hline$(\alpha, \beta)$ & $K$ & 50 & 102 & 182 & 368 \\
\hline$(1.4,1.4)$ & $\mathrm{N}=1$ & $1.55 \mathrm{e}-02$ & $1.027 \mathrm{e}-02$ & $7.47 \mathrm{e}-03$ & $4.4 \mathrm{e}-03$ \\
$(1.9,1.9)$ & $\mathrm{N}=2$ & $5.75 \mathrm{e}-03$ & $3.47 \mathrm{e}-03$ & $2.35 \mathrm{e}-03$ & $1.38 \mathrm{e}-03$ \\
\hline
\end{tabular}

\section{Conclusions}

In this work, we developed and analyzed DG methods for solving the two dimensional fractional elliptic problems. The DG methods can be obtained by suitably choosing the numerical fluxes in the flux formulation 
(3.6)-(3.8) have been shown (being like choosing the numerical fluxes in the classic problems). We made clear the relation between conservativity and consistency properties of the numerical fluxes and consistency and adjoint consistency properties of the primal formulation. We also have, theoretically and numerically, demonstrated an optimal order of convergence of $N+1$, when using LDG, IP and central fluxes. Compared to the condition number of the LDG, the central and the IP of discretized matrices, we showed that the LDG method leads to a much sparser operator in all cases and the IP method appears to offer a suitable compromise between LDG and central methods. 


\section{References}

[1] V. E. Tarasov, Fractional dynamics: applications of fractional calculus to dynamics of particles, fields and media, Springer Science \& Business Media, 2011.

[2] H. K. Moffatt, G. Zaslavsky, P. Comte, M. Tabor, Topological aspects of the dynamics of fluids and plasmas, volume 218, Springer Science \& Business Media, 2013.

[3] G. M. Zaslavsky, Chaos, fractional kinetics, and anomalous transport, Physics Reports 371 (2002) 461-580.

[4] A. I. Saichev, G. M. Zaslavsky, Fractional kinetic equations: solutions and applications, Chaos: An Interdisciplinary Journal of Nonlinear Science 7 (1997) 753-764.

[5] G. Zaslavsky, M. Edelman, Weak mixing and anomalous kinetics along filamented surfaces, Chaos: An Interdisciplinary Journal of Nonlinear Science 11 (2001) 295-305.

[6] R. Metzler, J. Klafter, The restaurant at the end of the random walk: recent developments in the description of anomalous transport by fractional dynamics, Journal of Physics A: Mathematical and General 37 (2004) R161.

[7] F. Mainardi, Fractional calculus and waves in linear viscoelasticity: an introduction to mathematical models, World Scientific, 2010.

[8] K. B. Oldham, Fractional differential equations in electrochemistry, Advances in Engineering Software 41 (2010) 9-12.

[9] E. Cuesta, M. Kirane, S. A. Malik, Image structure preserving denoising using generalized fractional time integrals, Signal Processing 92 (2012) 553-563.

[10] A. A. Kilbas, H. M. Srivastava, J. J. Trujillo, Theory and Applications of Fractional Differential Equations, Volume 204 (North-Holland Mathematics Studies), Elsevier Science Inc., New York, NY, USA, 2006.

[11] I. Podlubny, Fractional differential equations: an introduction to fractional derivatives, fractional differential equations, to methods of their solution and some of their applications, volume 198, Academic press, 1998.

[12] A. Kilbas, O. Marichev, S. Samko, Fractional integral and derivatives (theory and applications), Gordon and Breach, Switzerland 1 (1993) 1.

[13] R. L. Magin, Fractional calculus in bioengineering, Begell House Redding, 2006.

[14] V. J. Ervin, N. Heuer, J. P. Roop, Numerical approximation of a time dependent, nonlinear, space fractional diffusion equation, SIAM Journal on Numerical Analysis 45 (2007) 572-591. 
[15] W. Deng, Finite element method for the space and time fractional Fokker-Planck equation, SIAM Journal on Numerical Analysis 47 (2009) 204-226.

[16] C. Tadjeran, M. M. Meerschaert, A second-order accurate numerical method for the two-dimensional fractional diffusion equation, Journal of Computational Physics 220 (2007) 813-823.

[17] M. M. Meerschaert, C. Tadjeran, Finite difference approximations for fractional advection-dispersion flow equations, Journal of Computational and Applied Mathematics 172 (2004) 65-77.

[18] Z. zhong Sun, X. Wu, A fully discrete difference scheme for a diffusion-wave system, Applied Numerical Mathematics 56 (2006) 193-209.

[19] M. M. Meerschaert, H.-P. Scheffler, C. Tadjeran, Finite difference methods for two-dimensional fractional dispersion equation, Journal of Computational Physics 211 (2006) 249-261.

[20] M. Zayernouri, G. E. Karniadakis, Exponentially accurate spectral and spectral element methods for fractional ODEs, Journal of Computational Physics 257, Part A (2014) 460-480.

[21] X. Li, C. Xu, A space-time spectral method for the time fractional diffusion equation, SIAM Journal on Numerical Analysis 47 (2009) 2108-2131.

[22] T. Aboelenen, S. Bakr, H. El-Hawary, Fractional Laguerre spectral methods and their applications to fractional differential equations on unbounded domain, International Journal of Computer Mathematics (2015) 1-27.

[23] M. Zayernouri, G. E. Karniadakis, Fractional Sturm-Liouville eigen-problems: Theory and numerical approximation, Journal of Computational Physics 252 (2013) 495-517.

[24] K. Mustapha, W. McLean, Piecewise-linear, discontinuous Galerkin method for a fractional diffusion equation, Numerical Algorithms 56 (2011) 159-184.

[25] K. Mustapha, W. McLean, Uniform convergence for a discontinuous Galerkin, time-stepping method applied to a fractional diffusion equation, IMA Journal of Numerical Analysis 32 (2012) 906-925.

[26] K. Mustapha, W. McLean, Superconvergence of a discontinuous Galerkin method for fractional diffusion and wave equations, SIAM Journal on Numerical Analysis 51 (2013) 491-515.

[27] W. Deng, J. S. Hesthaven, Local discontinuous Galerkin methods for fractional diffusion equations, ESAIM: Mathematical Modelling and Numerical Analysis 47 (2013) 1845-1864.

[28] Q. Xu, J. S. Hesthaven, Discontinuous Galerkin method for fractional convection-diffusion equations, SIAM Journal on Numerical Analysis 52 (2014) 405-423. 
[29] L. Qiu, W. Deng, J. S. Hesthaven, Nodal discontinuous Galerkin methods for fractional diffusion equations on 2D domain with triangular meshes, Journal of Computational Physics 298 (2015) 678-694.

[30] T. Aboelenen, A high-order nodal discontinuous Galerkin method for nonlinear fractional Schrödinger type equations, Communications in Nonlinear Science and Numerical Simulation 54 (2018) 428 - 452.

[31] T. Aboelenen, Local discontinuous Galerkin method for distributed-order time and space-fractional convection-diffusion and Schrödinger type equations, Nonlinear dynamics (2017) 1-19.

[32] B. Jin, R. Lazarov, J. Pasciak, Z. Zhou, Error analysis of a finite element method for the space-fractional parabolic equation, SIAM Journal on Numerical Analysis 52 (2014) 2272-2294.

[33] H. Wang, D. Yang, Wellposedness of variable-coefficient conservative fractional elliptic differential equations, SIAM Journal on Numerical Analysis 51 (2013) 1088-1107.

[34] E. Kharazmi, M. Zayernouri, G. E. Karniadakis, A Petrov-Galerkin spectral element method for fractional elliptic problems, Computer Methods in Applied Mechanics and Engineering 324 (2017) 512-536.

[35] F. Bassi, S. Rebay, A high-order accurate discontinuous finite element method for the numerical solution of the compressible Navier-Stokes equations, Journal of computational physics 131 (1997) 267-279.

[36] B. Cockburn, C.-W. Shu, TVB Runge-Kutta local projection discontinuous Galerkin finite element method for conservation laws. II. General framework, Mathematics of computation 52 (1989) 411-435.

[37] B. Cockburn, C. Dawson, Approximation of the velocity by coupling discontinuous Galerkin and mixed finite element methods for flow problems, Computational Geosciences 6 (2002) 505-522.

[38] B. Cockburn, G. Kanschat, D. Schötzau, A locally conservative LDG method for the incompressible NavierStokes equations, Mathematics of Computation 74 (2005) 1067-1095.

[39] T. Aboelenen, A direct discontinuous Galerkin method for fractional convection-diffusion and Schrödinger type equations, arXiv:1708.04546 (2017).

[40] T. Aboelenen, H. El-Hawary, A high-order nodal discontinuous Galerkin method for a linearized fractional Cahn-Hilliard equation, Computers \& Mathematics with Applications 73 (2017) 1197-1217.

[41] X. Ji, H. Tang, High-order accurate Runge-Kutta (local) discontinuous Galerkin methods for one-and twodimensional fractional diffusion equations, Numerical Mathematics: Theory, Methods and Applications 5 (2012) 333-358.

[42] L. Qiu, W. Deng, J. S. Hesthaven, Nodal discontinuous Galerkin methods for fractional diffusion equations on 2D domain with triangular meshes, Journal of Computational Physics 298 (2015) 678-694. 
[43] D. N. Arnold, An interior penalty finite element method with discontinuous elements, SIAM journal on numerical analysis 19 (1982) 742-760.

[44] G. A. Baker, Finite element methods for elliptic equations using nonconforming elements, Mathematics of Computation 31 (1977) 45-59.

[45] C. E. Baumann, J. T. Oden, A discontinuous hp finite element method for convectiondiffusion problems, Computer Methods in Applied Mechanics and Engineering 175 (1999) 311-341.

[46] J. T. Oden, I. Babuŝka, C. E. Baumann, A discontinuous hp finite element method for diffusion problems, Journal of computational physics 146 (1998) 491-519.

[47] M. F. Wheeler, An elliptic collocation-finite element method with interior penalties, SIAM Journal on Numerical Analysis 15 (1978) 152-161.

[48] B. Rivière, M. F. Wheeler, V. Girault, Improved energy estimates for interior penalty, constrained and discontinuous Galerkin methods for elliptic problems. Part I, Computational Geosciences 3 (1999) 337-360.

[49] B. Rivière, M. F. Wheeler, V. Girault, A priori error estimates for finite element methods based on discontinuous approximation spaces for elliptic problems, SIAM Journal on Numerical Analysis 39 (2001) 902-931.

[50] D. N. Arnold, F. Brezzi, B. Cockburn, L. D. Marini, Unified analysis of discontinuous Galerkin methods for elliptic problems, SIAM journal on numerical analysis 39 (2002) 1749-1779.

[51] J. Peraire, P.-O. Persson, The compact discontinuous Galerkin (CDG) method for elliptic problems, SIAM Journal on Scientific Computing 30 (2008) 1806-1824.

[52] B. Cockburn, J. Gopalakrishnan, R. Lazarov, Unified hybridization of discontinuous Galerkin, mixed, and continuous Galerkin methods for second order elliptic problems, SIAM Journal on Numerical Analysis 47 (2009) 1319-1365.

[53] S. C. Brenner, L. Owens, L.-Y. Sung, A weakly over-penalized symmetric interior penalty method, Electron. Trans. Numer. Anal 30 (2008) 107-127.

[54] J. Wang, X. Ye, A weak Galerkin finite element method for second-order elliptic problems, Journal of Computational and Applied Mathematics 241 (2013) 103-115.

[55] K. Miller, B. Ross, An Introduction to the Fractional Calculus and Fractional Differential Equations, Wiley, 1993. URL: https://books.google.co.in/books?id=MOp_QgAACAAJ. 
[56] B. Cockburn, High-Order Methods for Computational Physics, Springer Berlin Heidelberg, Berlin, Heidelberg, 1999, pp. 69-224. URL: http://dx.doi.org/10.1007/978-3-662-03882-6_2, doi:10.1007/ 978-3-662-03882-6_2.

[57] P. Castillo, An optimal estimate for the local discontinuous Galerkin method, in: Discontinuous Galerkin methods, Springer, 2000, pp. 285-290.

[58] D. N. Arnold, F. Brezzi, B. Cockburn, D. Marini, Discontinuous Galerkin methods for elliptic problems, Lecture notes in computational science and engineering 11 (2000) 89-102.

[59] P. Castillo, B. Cockburn, I. Perugia, D. Schötzau, An a priori error analysis of the local discontinuous galerkin method for elliptic problems, SIAM Journal on Numerical Analysis 38 (2000) 1676-1706.

[60] J. S. Hesthaven, T. Warburton, Nodal discontinuous Galerkin methods: algorithms, analysis, and applications, Springer Science \& Business Media, 2007.

[61] R. A. Adams, Sobolev spaces, Academic Press, New York, 1975. 